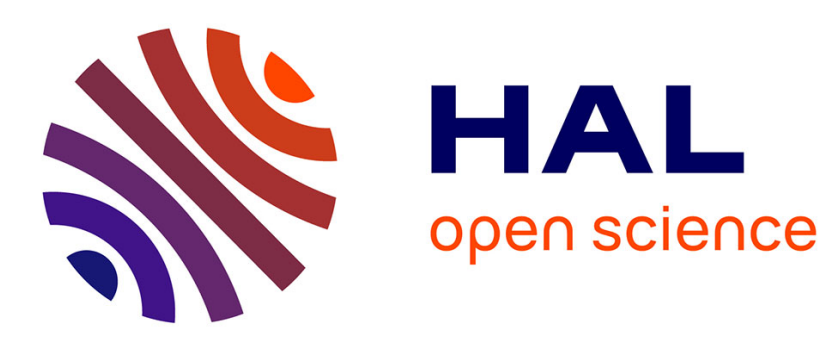

\title{
A globally convergent numerical algorithm for damaging elasto-plasticity based on the Multiplier method \\ Loredana Contrafatto, Massimo Cuomo
}

\section{To cite this version:}

Loredana Contrafatto, Massimo Cuomo. A globally convergent numerical algorithm for damaging elasto-plasticity based on the Multiplier method. International Journal for Numerical Methods in Engineering, 2005, 63 (8), pp.1089-1125. hal-00878215

\section{HAL Id: hal-00878215 \\ https://hal.science/hal-00878215}

Submitted on 29 Oct 2013

HAL is a multi-disciplinary open access archive for the deposit and dissemination of scientific research documents, whether they are published or not. The documents may come from teaching and research institutions in France or abroad, or from public or private research centers.
L'archive ouverte pluridisciplinaire HAL, est destinée au dépôt et à la diffusion de documents scientifiques de niveau recherche, publiés ou non, émanant des établissements d'enseignement et de recherche français ou étrangers, des laboratoires publics ou privés. 


\title{
A globally convergent numerical algorithm for damaging elasto-plasticity based on the Multiplier method
}

\author{
L. Contrafatto $\frac{\dot{s}}{*}$ and M. Cuomo*, \\ Deparment of Civil and Environmental Engineering, University of Catania, v.le A. Doria, 6, \\ 95125 Catania, Italy
}

\begin{abstract}
SUMMARY
In the paper it is proposed a new method for the solution of equilibrium problems based on a F.E. displacement formulation, that, at least in principle, is globally convergent as opposed to the classical Lagrangian method that presents only local convergence. The method, which appears to be particularly useful for plasticity models characterized by yield surfaces with regions of sharp curvature or corner points, is based on the Multiplier method. The structure of the procedure is presented and the consequent constrained optimization scheme is implemented for the case of associated plasticity coupled with damage. The main aspect of originality of the proposal is that it is not applied to the 'retum algorithm', but to entire equilibrium iteration.

At first, the local convergence properties of the constitutive equations are examined at the Gauss point level. It is proved that, also for involved constitutive models (a generalized Ottosen yield surface including isotropic hardening and damage is used in the applications), the convergence of a classical Newton's scheme is always reached with few iterations, ensuring a quadratic rate of convergence in the solution, provided a conversion of the inequality plastic constraint into an equality one is introduced, using an augmented Lagrangian functional for exactly evaluating the slack constraints. However, it is observed that the converged stresses are often attracted far from the initial trial point, towards regions with sharper curvature, and the main reason for the lack of convergence of the procedure is found in a divergence of the solution of the non-linear equilibrium equations. It is shown that the Multiplier method allows to enlarge the radius of convergence with respect to classical iterations based on the Lagrangian method. The price for the enlargement of the convergence radius is a higher number of iterations, since the Multiplier method presents only a linear rate of convergence. Indeed, the exact fulfilment of the compatibility and admissibility equations is not attained simultaneously, once an equilibrated solution is reached, but it is iterative. In the closure of the paper a convergence analysis of an elastic-plastic problem characterized by a yield criterion resulting from the convex hull of crises surfaces, and as a consequence, presenting regions of non-differentiability, is presented. It is shown how the ability of the Multiplier method in finding the solution of the structural problem for large loading step with respect to the classical Lagrangian technique compensate its slower convergence rate.
\end{abstract}

KEY WORDS: Lagrangian method; Multiplier method; plasticity; damage

\footnotetext{
"Correspondence to: Massimo Cuomo, Department of Civil and Environmental Engineering, University of Catania, vle A. Doria, 6, 95125 Catania, Italy.

${ }^{\dagger} \mathrm{E}$-mail: 1contra@dica.unict.it

${ }^{\ddagger}$ E-mail: mcuomo@ dica.unict.it
} 


\section{INTRODUCTION}

The incremental analysis of elastic-plastic type discretized problems within the context of displacement methods, at least for local constitutive models, is performed through a series of constrained non-linear optimization problems, corresponding to the numerical integration of the constitutive equations, performed at local level and to the iterative solution of the nonlinear equilibrium conditions. For a generic non-linear problem the two steps derive from the optimality conditions of a mixed energy functional, and specifically the local iteration corresponds to evaluate the elastic-plastic energy potential. Most of such iterative schemes are based on the Lagrangian method whose solution is sought using a Newton's procedure. It is well-known that it is conditionally convergent. When the initial approximation of the solution is close enough to the final solution, it exhibits a quadratic rate of convergence (local convergence) [1]. However the optimal convergence properties are lost when the structure of the problem is not 'sufficiently convex' in the vicinity of the solution point, or when the predictor is not within the radius of convergence of the method. This often happens in conjunction with involved constitutive models, or in the neighbourhood of corner points of the constraint domain, situation often met when generalized coupled plasticity-damage models are used. Severe conditions are also encountered in many constitutive equations proposed for concrete, either because the yield domain presents regions of sharp curvature (MohrCoulomb, Ottosen models etc.) [2], or because of the introduction of energy functionals with discontinuous second derivatives (bimodular materials) [3]. A wide number of alternatives have been proposed [4-7], that employ either combined secant/tangent strategies [8] or ad hoc switching rules between Newton's steps and globally convergent algorithms, usually based on line searches [4], which, however, are known to be very sensitive to the search parameters. In most of the studies concerning convergence properties of FE algorithms, the attention has been focused on the performance of the so-called 'return algorithm', that is what we have defined as local problem, in the implicit hypothesis that failure of the procedure can only occur if an admissible solution cannot be found. However, it appears that there are cases when, although a locally admissible stress state is reached, the algorithm diverges in finding an equilibrate solution. This happens when the admissible stress state, or the normal to the plastic potential function, is quite different from the predicted values, as typically occurs near corner points of the yield domain, or for involved plasticity conditions. In these cases, in order to achieve convergence, it is necessary to reduce the step-size.

In this paper the Lagrangian method employed for the iterative solution of non-linear elasticplastic problems is firstly reviewed, and it will be shown that a proper implementation of the method, with slight modifications with respect to the classical scheme, allows for a significant improvement of its convergence properties. This is achieved simply substituting the inequality constraints with equivalent equality constraints, in the form described in Section 2. In doing that, an arbitrary real positive constant is introduced in their expression, whose value does not affect the convergence of the problem.

Then an alternative to the Lagrangian method is proposed that falls within the class of the so-called 'Multiplier methods'. It allows to enlarge the convergence radius, although, at least in principle, at the cost of a slower convergence rate [9]. The specific application of the method and the numerical algorithm will be described in detail in the subsequent sections. Its main difference with the Lagrangian methods is that the exact fulfilment of the constitutive equations is not enforced once an iterative equilibrated solution is reached; rather 
also the satisfaction of the admissibility equations is iterative. The method presents a linear or super-linear convergence rate, according to its implementation, so that one should expect some loss of efficiency of the method. However, for the class of problems described, the possibility of increasing the step-size and the greater attitude to convergence in the proximity of points of sharp curvature, compensate for the theoretically slower rate of convergence. Of course, an efficient strategy of solution should include a switching rule for turning from Lagrangian to Multiplier methods only when severe convergence conditions are met. However, this coupled strategy will not be discussed in the paper, that will only concentrate on a comparison between the methods described. Recently an application of the Multiplier method to plasticity problems has been proposed by Pérez-Foguet and Armero [4] and Pérez-Foguet et al. [5], who denoted it as 'dual closed point projection method'. However, apart from differences in the detailed implementation of the procedure, they applied the method only to the local admissibility problem. In the present paper the Multiplier method is applied directly to the mixed Hellinger-Reissner functional governing the elastic-plastic problem, that is it affects the entire equilibrium iterations. The originality of the proposal is clear in the description of its implementation given in Section 3.2. The efficiency of the proposed strategy will be shown with reference to very simple equilibrium problems of elastic-plastic bodies. Either the perfect plasticity and the hardening cases will be examined. Furthermore, the implementation of the method, thanks to the use of a robust constitutive model, allows a straightforward extension to coupled damaging models.

The outline of the paper is as follows. In Sections 2 and 3 the Lagrangian method for elastic-plastic problems is discussed; then the implementation of the method of the multipliers is presented and commented upon. The efficiency of the algorithm will first be discussed with reference to the strain driven problems (local iterations) in Section 4.1.1, then the force driven problems will be considered in Section 4.1.2, for which also the equilibrium conditions must be imposed iteratively. Finally, in Section 4.2 the two methods illustrated in the paper will be applied to a rather complex load process for an elastic-plastic-damaging material, comparing the maximum step-size length that can be reached with the different methods. After the conclusions an appendix dedicated to the use of augmented Lagrangian functions in the calculation of the elastic-plastic potential is added for completeness.

\section{OPTIMIZATION ALGORITHM FOR ONE-SIDED INEQUALITY CONSTRAINT}

An inequality constrained optimization problem is defined as

$$
\begin{array}{ll}
\text { minimize } & f(x) \\
\text { subject to } & g(x) \leqslant 0
\end{array}
$$

One possible strategy for solving the problem is the conversion of the inequality problem into an equality constrained one, defined as

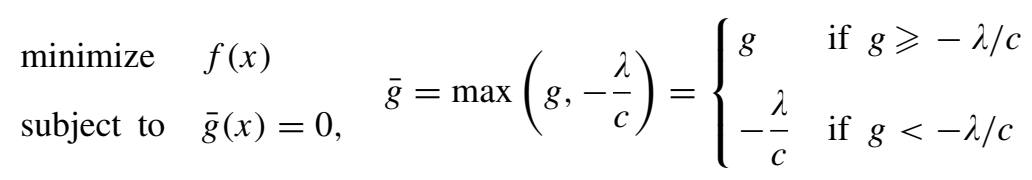


where $c$ is any positive real constant and $\lambda$ is the Lagrangian multiplier of the constraint $\bar{g}$. The conversion is obtained introducing slack variables and performing a closed form minimization of the augmented Lagrangian functional associated to problem (1) [9]

$$
L_{c}(x, z, \lambda, c)=f(x)+\lambda\left[g(x)+z^{2}\right]+\frac{1}{2} c\left[g(x)+z^{2}\right]^{2}
$$

In fact, the optimality conditions of $L_{c}$ coincide with the solution $x^{*}$ of the equality constrained problem

$$
\begin{array}{ll}
\operatorname{minimize} & f(x) \\
\text { subject to } & g(x)+z^{2}=0, \quad z \in R
\end{array}
$$

This is equivalent to problem (1). The minimization of (3) w.r.t. $z^{2}$ yields the condition

$$
z^{2}=-\left[\frac{\lambda}{c}+g(x)\right]
$$

so that, in order for $z^{2}$ to be non-negative, it must be

$$
z^{2}=\max \left\{0,-\left[\frac{\lambda}{c}+g(x)\right]\right\}
$$

and, substituting (5) into the second of (4), the equivalent equality constrained problem (2) is obtained. Note that, thanks to the conversion of the inequality constraint into an equality one, the Lagrangian multiplier is not sign restricted. In (2) the choice of $c$ is little sensitive for the convergence of the solution algorithm, provided numerical ill-conditioning be avoided for extremely large or extremely small values of the parameter. In the sequel of the paper the inequality constrained problem (1) will be always substituted with the equivalent equality constrained problem (2), unless explicitly declared.

Two classes of solution methods for the equality constrained optimization problems are then examined, i.e. the Lagrangian method and the Multiplier method.

\subsection{Lagrangian method}

Lagrangian algorithms are formulated as optimization problems for the classical Lagrangian functional

$$
\min _{x} \max _{\lambda} L(x, \lambda), \quad L=f(x)+\lambda \bar{g}(x)
$$

obtained introducing a Lagrangian regularization of the constraint

$$
I_{K}^{(x)}=\left\{\begin{array}{ll}
0 & \text { if } x \in K, \\
+\infty & \text { if } x \notin K,
\end{array} \quad K=\{x: \bar{g}(x)=0\}\right.
$$

$K$ being the admissibility domain. It is easy to prove that $I_{K}^{(x)}=\sup _{\lambda}(\lambda \bar{g}(x))$.

The algorithm consists in evaluating a direction of descent for the unconstrained Lagrangian function $L(x, \lambda)$ simultaneously updating the primal and the dual variables. It is well known that the radius of convergence of this method is quite restricted, in the sense that it requires 
a good starting point for both $x$ and $\lambda$. The solution can be obtained using either a Newton's method, or a modified Newton's method, or a quasi Newton method (DFP, BFGS, etc.), whose general form is

$$
\left[\begin{array}{ll}
H_{x x} & H_{x \lambda} \\
H_{\lambda x} & H_{\lambda \lambda}
\end{array}\right]_{k}\left[\begin{array}{l}
\Delta x_{k} \\
\Delta \lambda_{k}
\end{array}\right]=-\left[\begin{array}{c}
\nabla_{x} L \\
\nabla_{\lambda} L
\end{array}\right]_{k}, \quad\left\{\begin{array}{l}
x_{k+1}=x_{k}+\Delta x_{k} \\
\lambda_{k+1}=\lambda_{k}+\Delta \lambda_{k}
\end{array}\right.
$$

where $[H]_{k}$ is the Hessian matrix at the generic iteration. If a Newton's direction is used a quadratic rate of convergence is reached once the solution is sufficiently approached.

The present version of the Lagrangian method is different from the classical Lagrangian technique, applied directly to the inequality constrained problem, that does not employ transformation (2) and seeks the optimum of the functional

$$
L^{+}(x, \lambda)=f(x)+\lambda g^{+}(x), \quad g^{+}(x)=\max [g(x), 0]
$$

Note that, for inequality constraints, functional (6) presents an implicit dependence on the parameter $c$ that appears in the definition of $\bar{g}$. This aspect will be further commented in Section 3.

\subsection{Multiplier method}

The Multiplier method is based on the minimization of the augmented Lagrangian functional

$$
L_{c}(x, \lambda, c)=f(x)+\lambda \bar{g}(x)+\frac{1}{2} c \bar{g}(x)^{2}
$$

It is obtained by means of the addition of a penalty term to the Lagrangian functional $L(x, \lambda)$, so that the special case $L_{0}=L_{c}(x, \lambda, 0)$ gives the Lagrangian functional defined in (6). Usually a classical quadratic penalty function is employed: this is generally sufficient to ensure that the augmented Lagrangian functional has a minimum for fixed values of the pair $(\lambda, c)$.

The solution is found with a staggered procedure. First the value $x\left(\lambda_{k}, c_{k}\right)$ that minimizes $L_{c}$ keeping fixed the value of the multipliers is obtained; next the Lagrangian multipliers are updated by means of a 1 st order update formula [10,11] or a 2 nd order update formula [12]

$$
\begin{aligned}
& \lambda_{k+1}=\lambda_{k}+c_{k} \bar{g}\left[x\left(\lambda_{k}, c_{k}\right)\right] \quad \text { 1st order iteration } \\
& \lambda_{k+1}=\lambda_{k}+B_{k}^{-1} \bar{g}\left[x\left(\lambda_{k}, c_{k}\right)\right] \quad \text { 2nd order iteration, } \quad B_{k}=\nabla \bar{g}_{k}^{\mathrm{T}}\left(\nabla_{x, x}^{2} L_{c_{k}}\right)^{-1} \nabla \bar{g}_{k}
\end{aligned}
$$

The penalty parameter $c$ used in (9), in principle, could be different from the real positive constant appearing in the definition of $\bar{g}$. However, it can be shown that the best choice is to use the same value for both the constants. This can be easily understood with reference to the 1 st order iteration update. Indeed, in the case the constraint is satisfied, it results

$$
\bar{g}\left(x\left(\lambda_{k}, c_{k}\right)\right)=-\frac{\lambda_{k}}{c_{k}}
$$

so that formula (10a) automatically annihilates the corresponding Lagrangian multiplier. 
The method of the multipliers can rationally be interpreted as an optimization procedure for the so-called dual functional, given by

$$
D(\lambda)=\min _{x} L_{c}(x, \lambda, c)=f[x(\lambda, c)]+\lambda \bar{g}[x(\lambda, c)]+\frac{1}{2} c(\bar{g}[x(\lambda, c)])^{2}
$$

It can be shown that the optimality condition for (11) is

$$
\nabla D(\lambda)=\bar{g}[x(\lambda, c)]=0
$$

Therefore the method of the multipliers is equivalent to find the root $\lambda$ of Equation (12) if $x(\lambda, c)$ is the value of the primal variables that minimizes the augmented Lagrangian functional (9) w.r.t. $x$. In the language of Solid Mechanics, this means that the iterative updates of the Lagrangian multipliers tend to enforce plastic compatibility for equilibrated stress states.

A 1 st order update is equivalent to perform the steepest ascent step with constant step-size $c$ of the dual functional $D(\lambda)$, while a 2 nd order update is equivalent to a Newton-like iteration on the dual functional [13].

Since it is not possible to reach the exact minimization of the primal functional $L_{c}$, the procedure must include some tuning rule for the penalty parameter $c$ and, eventually, for the convergence tolerance. Therefore the problem is solved through the following steps:

$$
\begin{gathered}
\min _{x} L_{c}(x, \lambda, c) \\
\text { update } \lambda \\
\text { update } c
\end{gathered}
$$

It can be demonstrated that there exists a positive constant $\delta$ so that the Multiplier method converges to the optimal value $\left(x^{*}, \lambda^{*}\right)$ provided the pairs $\left(\lambda_{k}, c_{k}\right)$, including the initial values $\lambda_{0}, c_{0}$, are chosen in the region

$$
\left|\lambda_{k}-\lambda^{*}\right|<c_{k} \delta, \quad \delta>0, \quad \bar{c} \leqslant c_{k} \leqslant c_{k+1} \quad \forall k=0,1 \ldots
$$

$\bar{c}$ being a positive scalar such that $\nabla_{x, x}^{2} L_{c}\left(x^{*}, \lambda^{*}, \bar{c}\right)>0$.

The region of pairs $(\lambda, c)$ for which the Multiplier method is defined is shown in Figure 1 , where it is evident that a poor choice of $\lambda_{0}$ can be compensated by a considerably high value of $c_{0}$. In Reference [14] a strategy is proposed for the optimal selection of the initial penalty parameter $c_{0}$, based on the estimation of the ratio between the largest eigenvalue of the Hessian matrix of the function $f$ and of the constraint.

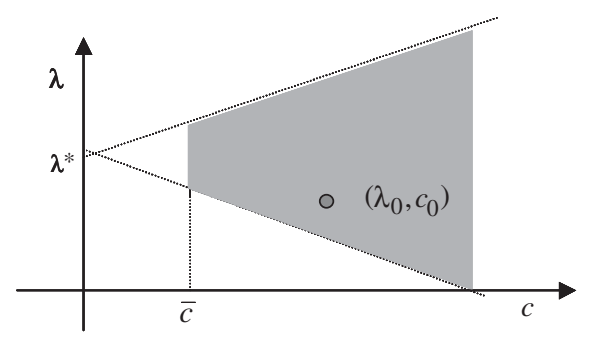

Figure 1. Convergence domain for pairs $(\lambda, c)$ in the Multiplier method. 
In order to enforce convergence, the penalty parameter $c$ should then be increased during the iterations. In practice, this is performed if a sufficient reduction in the constraint violation is not reached over the previous optimization step

$$
\begin{array}{ll}
c_{k+1}=\beta c_{k} & \text { if }|\bar{g}|_{k+1}>\gamma|\bar{g}|_{k} \\
c_{k+1}=c_{k} & \text { if }|\bar{g}|_{k+1} \leqslant \gamma|\bar{g}|_{k}
\end{array} \quad \text { for some } \beta>1 \text { and } 0<\gamma<1
$$

Typical values for the scalars $\beta$ and $\gamma$ are $\beta=10$ and $\gamma=0.25$. In Section 4 it will be shown that sometimes a different choice of the $c$ update scheme is beneficial.

An estimate for the minimum value of the scalar $\bar{c}$ necessary for (13) to be met is given by the condition that it has to be large enough to make the Hessian of the functional $L_{c}$ positive definite at the solution. It can be proved that

$$
\begin{array}{ll}
\nabla_{x, x}^{2} L_{c}\left(x^{*}, \lambda^{*}, \bar{c}\right)>0 & \Longleftrightarrow \bar{c}>\max \left\{-e_{1},-e_{2}, \ldots,-e_{n}\right\} \\
e_{i} \text { eigenvalue of } & \left\{\nabla_{x} \bar{g}^{\mathrm{T}}\left(x^{*}\right)\left[\nabla_{x, x}^{2} L_{c}\left(x^{*}, \lambda^{*}, \bar{c}\right)\right]^{-1} \nabla_{x} \bar{g}\left(x^{*}\right)\right\}^{-1}
\end{array}
$$

that is, $\bar{c}$ must be greater than the opposite of the minimum negative eigenvalue of the inverse matrix of the Hessian of the dual functional $D\left(\lambda^{*}\right)$. If all the eigenvalues $e_{i}$ are non-negative any positive $\bar{c}$ can be used as threshold level. In Structural Mechanics this condition is met if the problem is convex, i.e. if the internal energy and the plastic potential are convex.

\subsection{An academic example: the minimization of a simple function of two variables}

Let $f(x, y)$ be the simple function of two scalar variables defined as

$$
f(x, y)=\frac{1}{2}\left[\begin{array}{cc}
10 & \frac{1}{4} \\
\frac{1}{4} & \alpha
\end{array}\right]\left[\begin{array}{l}
x+4 \\
y-5
\end{array}\right] \cdot\left[\begin{array}{l}
x+4 \\
y-5
\end{array}\right]
$$

where $\alpha$ is a real parameter that rules the convexity of the function $f$, as it is shown in Figure 2. A value of $\alpha$ greater than $\frac{1}{160}$ makes the function $f$ convex. The smaller $\alpha$, the higher is the curvature of the function. If $\alpha$ is smaller than $\frac{1}{160}$, the function $f$ is a saddle functional in $(x, y)$.
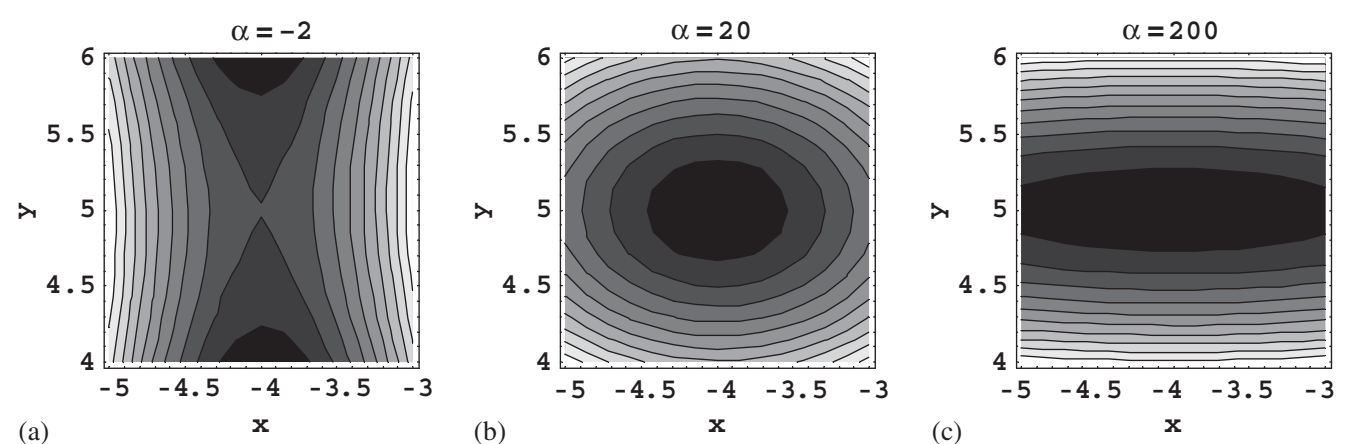

Figure 2. Contour levels of function $f(x, y)$ for different values of the parameter $\alpha$ : (a) $\alpha=-2$; (b) $\alpha=20$; and (c) $\alpha=200$. 
Table I. Convergence of algorithms 1,2,3 for problem (16).

\begin{tabular}{|c|c|c|c|c|c|}
\hline \multirow[b]{2}{*}{$c$} & \multicolumn{2}{|c|}{ Alg. 1} & \multicolumn{2}{|c|}{ Alg. 2} & \multirow[t]{2}{*}{ Alg. 3} \\
\hline & Direct & Dual & Direct & Dual & \\
\hline $10^{-7}$ & - & - & $\max 5-21$ & 7 & 7 \\
\hline $10^{-3}$ & - & - & $\max 5-21$ & 7 & 7 \\
\hline $10^{-2}$ & - & - & $\max 5-21$ & 7 & 7 \\
\hline $10^{-1}$ & - & - & $\max 3-22$ & 7 & 7 \\
\hline $10^{0}$ & $\max 3$ & $\sim 700$ & $\max 3-22$ & 7 & 7 \\
\hline $10^{1}$ & $\max 4-679$ & 464 & $\max 4-21$ & 6 & 7 \\
\hline $10^{2}$ & $\max 6-89$ & 55 & $\max 6-15$ & 5 & 7 \\
\hline $10^{3}$ & $\max 8-30$ & 12 & $\max 8-15$ & 4 & 7 \\
\hline $10^{4}$ & $\max 10-21$ & 6 & $\max 10-15$ & 3 & 7 \\
\hline
\end{tabular}

$\alpha=200$, constant penalty parameter, starting point $(-3,-1,0)$.

Consider the problem

$$
\begin{array}{ll}
\text { minimize } & f(x, y) \\
\text { subject to } & g(x, y) \leqslant 0, \quad g(x, y)=\sqrt{x^{2}+y^{2}-x y}-3
\end{array}
$$

It has been solved by means of the following algorithms:

1. Multiplier method with 1st order update (See Section 2.2, formula (10a)).

2. Multiplier method with 2nd order update (See Section 2.2, formula (10b)).

3. Lagrangian method applied to the functional $L$ (See Section 2.1, formula (6)).

A Newton's algorithm has been employed for finding the optimum value of $(x, y)$ in the Multiplier methods and of $(x, y, \lambda)$ in the Lagrangian method.

Two different values of the parameter $\alpha$ have been considered: $\alpha=200$ and -2 .

The results are reported in Tables I-III, for an initial penalty parameter in the range $10^{-7} \leqslant c \leqslant 10^{4}$. In them, for each of the Multiplier algorithms 1 and 2 , the first column reports the maximum number of iterations needed for finding the optimum value of the direct variables $(x, y)$ in a single minimization (that generally occurs at the first step) and the total number of direct iterations; the second column reports the number of dual iterations, i.e. of multiplier updates. For the Lagrangian algorithm 3 the number of Newton's step is reported.

Tables I and II refer to $\alpha=200$. In this case the constrained minimum is located at the point $(x, y, \lambda)=(1.24732,3.42244,336.791)$, as it is shown in Figure 3 . The results obtained keeping $c$ fixed at its intial value $c_{0}$, are illustrated in Table I. For small values of the initial penalty parameter $c_{0}$, no convergence is achieved using the Multiplier algorithm 1 (1st order Hestenes-Powell formula). The Multiplier method with 2nd order Buys update is convergent for a wide range of $c$ values and for higher values of $c_{0}$, a considerable reduction in the number of iterations is observed. The Lagrangian method is always convergent; as it is evident from the results reported in the table, the choice of $c$, that influences $\bar{g}$, does not affect the rate of convergence of the Lagrangian algorithm 3.

Table II summarizes the results for $\alpha=200$ using the update formula (14) with $\beta=10$ and $\gamma=0.25$. The column $c_{0}$ reports the initial value of the penalty parameter. A considerable 
Table II. Convergence of algorithms 1,2,3 for problem (16).

\begin{tabular}{lcccccc}
\hline & \multicolumn{2}{c}{ Alg. 1 } & & \multicolumn{2}{c}{ Alg. 2} & \multirow{2}{*}{ Alg. 3 } \\
\cline { 2 - 3 }$c_{0}$ & Direct & Dual & & Direct & Dual & \\
\hline $10^{-7}$ & $\max 4-45$ & 21 & & $\max 5-21$ & 7 & 7 \\
$10^{-3}$ & $\max 4-41$ & 17 & & $\max 5-21$ & 7 & 7 \\
$10^{-2}$ & $\max 4-40$ & 16 & & $\max 5-21$ & 7 & 7 \\
$10^{-1}$ & $\max 4-39$ & 15 & & $\max 5-21$ & 6 & 7 \\
$10^{0}$ & $\max 3-37$ & 14 & & $\max 4-19$ & 6 & 7 \\
$10^{1}$ & $\max 5-24$ & 6 & & $\max 5-20$ & 5 & 7 \\
$10^{2}$ & $\max 6-21$ & 5 & & $\max 6-17$ & 4 & 7 \\
$10^{3}$ & $\max 8-18$ & 4 & & $\max 8-16$ & 3 & 7 \\
$10^{4}$ & $\max 10-18$ & 4 & & $\max 10-15$ & 3 & 7 \\
\hline
\end{tabular}

$\alpha=200$, penalty parameter update, starting point $(-3,-1,0)$.

Table III. Convergence of algorithms 1,2,3 for problem (16).

\begin{tabular}{lcccccc}
\hline & \multicolumn{2}{c}{ Alg. 1 } & & \multicolumn{2}{c}{ Alg. 2 } & \multirow{2}{*}{ Alg. 3 } \\
\cline { 2 - 3 }$c_{0}$ & Direct & Dual & & Direct & Dual & \\
\hline $10^{-7}$ & $\max 5-29$ & 13 & & n.c. & - & n.c. \\
$10^{-3}$ & $\max 5-25$ & 9 & & n.c. & - & n.c. \\
$10^{-2}$ & $\max 5-23$ & 7 & & n.c. & - & n.c. \\
$10^{-1}$ & $\max 5-21$ & 6 & & n.c. & - & n.c. \\
$10^{0}$ & $\max 5-19$ & 5 & & $\max 5-20$ & 5 & n.c. \\
$10^{1}$ & $\max 7-19$ & 4 & & $\max 7-19$ & 4 & n.c. \\
$10^{2}$ & $\max 9-21$ & 5 & & $\max 9-17$ & 3 & 23 \\
$10^{3}$ & $\max 10-17$ & 3 & & $\max 10-16$ & 3 & 23 \\
$10^{4}$ & $\max 10-15$ & 3 & & $\max 10-14$ & 2 & 23 \\
\hline
\end{tabular}

$\alpha=-2$, penalty parameter update, starting point $(-10,10,0)$.
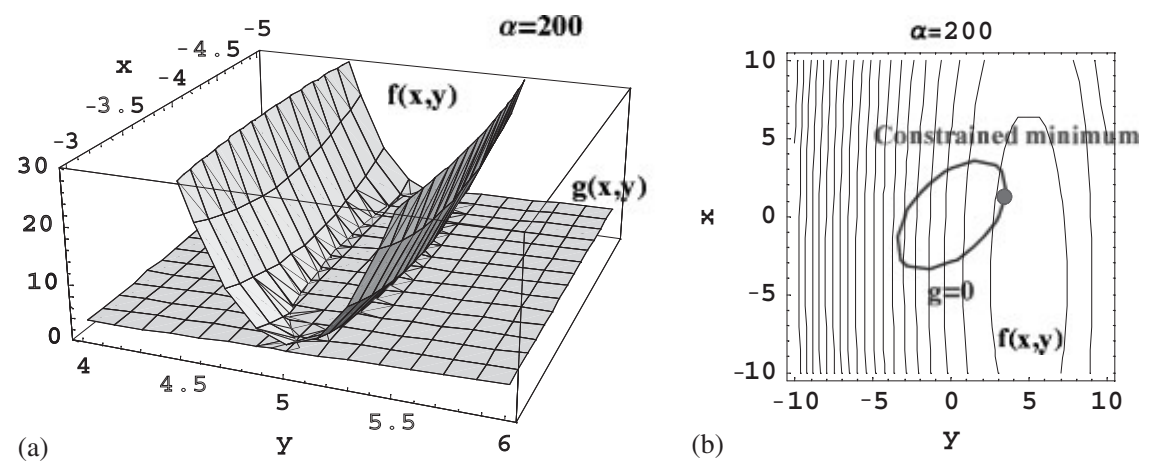

Figure 3. Constrained minimum of the convex function $f(x, y)$ for $\alpha=200$. 


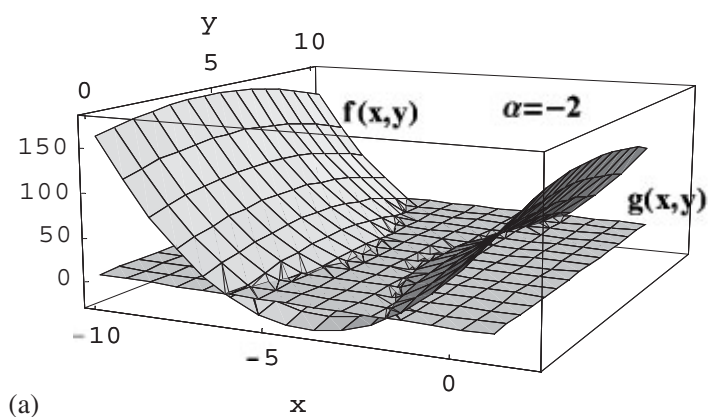

(a)

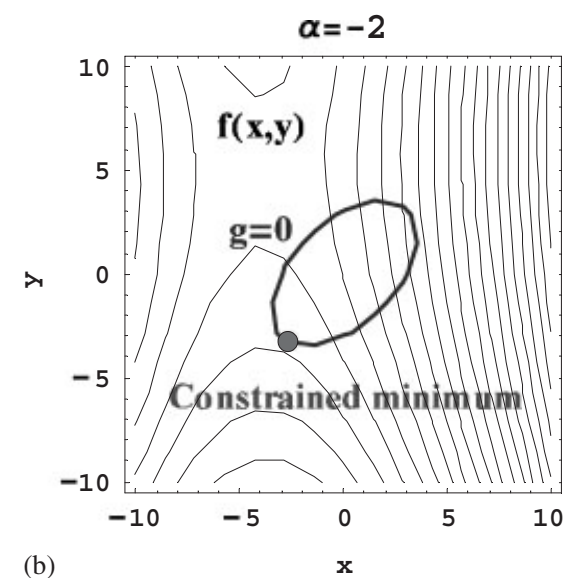

(b)

Figure 4. Constrained minimum of the saddle function $f(x, y)$ for $\alpha=-2$.

Table IV. Convergence of algorithms 1,2,3 for problem (16).

\begin{tabular}{lcclcrr}
\hline & \multicolumn{2}{c}{ Alg. 1 } & & \multicolumn{2}{c}{ Alg. 2 } & Alg. 3 \\
\cline { 2 - 3 }$\left(x_{0}, y_{0}\right)$ & Direct & Dual & & Direct & Dual & \\
\hline$(-10,10)$ & $\max 10-15$ & 3 & & $\max 10-14$ & 2 & 23 \\
$(0,10)$ & $\max 11-16$ & 3 & & $\max 11-15$ & 2 & 7 \\
$(10,10)$ & $\max 11-16$ & 3 & & $\max 11-15$ & 2 & 46 \\
$(20,10)$ & $\max 11-16$ & 3 & & $\max 11-15$ & 2 & 36 \\
$(30,10)$ & $\max 11-16$ & 3 & & $\max 11-15$ & 2 & 24 \\
\hline
\end{tabular}

$\alpha=-2$, initial penalty parameter $c_{0}=10^{4}$, different starting points $\left(x_{0}, y_{0}, 0\right)$.

improvement of the convergence is obtained for the Multiplier method, especially with the 1 st order update (algorithm 1). Of course, no difference is observed for the Lagrangian algorithm.

Table III refers to $\alpha=-2$. In this case the function $f(x, y)$ presents a saddle point: it is convex w.r.t the variable $x$ and concave w.r.t. $y$. The constrained minimum is located at the point $(x, y, \lambda)=(-2.74682,-3.20132,27.4346)$, as shown in Figure 4 .

The choice of the trial point strongly influences the rate of convergence. Table III reports the results considering the penalty parameter update based on Equation (14) with $\beta=10, \gamma=0.25$ and starting point $(x, y, \lambda)=(-10,10,0)$. It appears that the Multiplier method 1 is able to reach the solution point of the problem in the entire range of investigated $c$ with a modest number of iterations, especially when the initial penalty parameter $c_{0}$ is kept greater than $10^{2}$. Conversely, convergence is lost for low values of $c_{0}$ using 2 nd order algorithms, i.e. Multiplier method with Buys update. The Lagrangian method presents in this case a slower convergence. Furthermore, the value of the parameter $c$ becomes more important.

This observation is strengthened by the results of Table IV, obtained solving the same problem starting with different trial points, in which case the Lagrangian method presents a different rate of convergence, depending on the proximity of the trial point, while the rate of convergence of the Multiplier algorithms is insensitive to the initial choice of the predictor point. 
The procedures illustrated in the previous section are now applied to solve a structural problem in the context of a displacement formulation [15]. The constitutive behaviour is assumed to be elastic-plastic coupled with isotropic damage and hardening. The state of the system is defined by means of conjugated pairs of variables, posed in separating duality by bilinear forms that represent the internal virtual work

$$
\eta=\left[\begin{array}{c}
\varepsilon \\
\alpha \\
\omega
\end{array}\right] \begin{gathered}
\text { strain } \\
\text { internal dislocation } \\
\text { damage variable }
\end{gathered}=\left[\begin{array}{c}
\sigma \\
\chi \\
\zeta
\end{array}\right] \begin{gathered}
\text { stress } \\
\text { hardening forces } \\
\text { damage work }
\end{gathered}
$$

In the structural problem linear compatibility equations are considered

$$
C_{\eta} u=\eta, \quad \eta=(\varepsilon, \alpha, \omega), \quad C_{\eta}=\left[\begin{array}{lll}
C & 0 & 0
\end{array}\right]
$$

$u$ being the displacements and $C$ the equilibrium operator.

Each kinematic variable is (additively) decomposed into the sum of a reversible and an irreversible component, that enter as arguments, respectively, in the stored energy functional $e\left(\varepsilon_{\mathrm{e}}, \alpha_{\mathrm{e}}, \omega_{\mathrm{e}}\right)$ and in the dissipation functional $d\left(\dot{\varepsilon}_{\mathrm{p}}, \dot{\alpha}_{\mathrm{p}}, \dot{\omega}_{\mathrm{p}}\right)$. Denoting by $\eta=\left(\eta_{\mathrm{e}}+\eta_{\mathrm{p}}\right)$ the whole set of kinematic variables, and by $\tau$ the dual static variables, under mild hypotheses of sub-differentiability, it is

$$
\tau \in \partial_{\eta_{\mathrm{e}}} e, \quad \tau \in \partial_{\dot{\eta}_{\mathrm{p}}} d, \quad \eta_{\mathrm{e}} \in \partial_{\tau} e^{\mathrm{c}}, \quad \dot{\eta}_{\mathrm{p}} \in \partial_{\tau} d^{\mathrm{c}}
$$

where the apex $c$ denotes the conjugate potential in the sense of a Legendre transformation

$$
e^{\mathrm{c}}(\tau)=\sup _{\eta_{\mathrm{e}}}\left\{\left\langle\tau, \eta_{\mathrm{e}}\right\rangle-e\left(\eta_{\mathrm{e}}\right)\right\}, \quad d^{\mathrm{c}}(\tau)=\sup _{\eta_{\mathrm{p}}}\left\{\left\langle\tau, \dot{\eta}_{\mathrm{p}}\right\rangle-d\left(\dot{\eta}_{\mathrm{p}}\right)\right\}
$$

From here on, brackets $\langle\cdot, \cdot\rangle$ will stand for the relevant scalar product between conjugate pairs of variables.

The stored energy functional $e\left(\eta_{\mathrm{e}}\right)$ is non-convex for damage constitutive equations, and is twice differentiable almost everywhere, with discontinuous 2 nd derivative only on a domain of zero measure. On the contrary, it is assumed that the dissipation potential is a convex subdifferentiable functional. If it is an additive sub-linear function, homogeneous of degree one, a generalized associative elastic-plastic-type constitutive model with no time scale is obtained [15]

$$
d\left(\dot{\eta}_{\mathrm{p}}\right)=\operatorname{supp} K, \quad d^{\mathrm{c}}(\tau)=\operatorname{ind} K
$$

As a consequence, the inelastic model is associative and is based on a single dissipation potential

$$
\dot{\eta}_{\mathrm{p}} \in \partial_{\tau} d^{\mathrm{c}}(\tau)=\lambda \partial g(\tau), \quad \lambda \in \partial R^{-}(g)
$$

In (21) the convex yield function $g(\tau)$ has been introduced, such that

$$
K=\{\tau: g(\tau) \leqslant 0\}
$$


For multiple dissipation modes, the domain $K$ is defined by more than one yield function, so that

$$
g(\tau)=\sup _{i}\left\{g_{i}(\tau)\right\}
$$

A major consequence of the above hypotheses is that the Hessian matrix of the local system of the compatibility and admissibility equations is not always positive definite.

For the sake of simplicity, the following treatment will refer to the case of a convex internal energy functional. However, the algorithms and the conclusions apply also to the case of nonconvex energy functionals, although it cannot be regarded as an optimization procedure. In the hypotheses stated, the solution of the structural problem consists in optimizing the 2-fields generalized Hellinger-Reissner functional

$$
\begin{gathered}
\inf _{u} \sup _{\tau} \Omega_{R}(u, \tau) \\
\Omega_{R}(u, \tau)=\left\langle\tau, C_{\eta} u\right\rangle-\langle f, u\rangle-e^{\mathrm{c}}(\tau)-d^{\mathrm{c}}(\tau) \Delta t-\left\langle\tau, \eta_{\mathrm{p}}^{0}\right\rangle
\end{gathered}
$$

Therefore, at each load step the solution point for the problem has to be found

$$
\begin{gathered}
\inf _{u}\left\{\Phi_{\mathrm{ep}}\left(C_{\eta} u\right)-\langle f, u\rangle\right\} \\
\Phi_{\mathrm{ep}}=\sup _{\tau}\left\{\left\langle\tau, C_{\eta} u\right\rangle-e^{\mathrm{c}}(\tau)-d^{\mathrm{c}}(\tau) \Delta t-\left\langle\tau, \eta_{\mathrm{p}}^{0}\right\rangle\right\}
\end{gathered}
$$

In (25) the first problem corresponds to the equilibrium equations, $\Phi_{\mathrm{ep}}\left(C_{\eta} u\right)$ being the elastic-plastic tangent operator; the second problem enforces compatibility conditions at local level.

\subsection{Lagrangian method}

The complementary dissipation functional (20) is regularized by

$$
\begin{aligned}
& d^{\mathrm{c}}(\tau)=\sup _{\lambda \in R^{+}}\{\lambda g(\tau)\}=\sup _{\lambda}\{\lambda \bar{g}(\tau)\}, \quad \bar{g}(\tau)=\sup _{i}\left\{\bar{g}_{i}(\tau)\right\} \\
& \bar{g}_{i}(\tau)=\max \left(g_{i},-\frac{\lambda_{i}}{c}\right), \quad c \in R^{+}
\end{aligned}
$$

so that $(25)$ becomes

$$
\begin{gathered}
\inf _{u}\left\{\Phi_{\mathrm{ep}}\left(C_{\eta} u\right)-\langle f, u\rangle\right\} \\
\Phi_{\mathrm{ep}}=\sup _{\tau} \inf _{\lambda}\left\{\left\langle\tau, C_{\eta} u\right\rangle-e^{\mathrm{c}}(\tau)-\lambda \bar{g}(\tau)-\left\langle\tau, \eta_{\mathrm{p}}^{0}\right\rangle\right\} \\
=\sup _{\tau}\left\{\left\langle\tau, C_{\eta} u\right\rangle-e^{\mathrm{c}}(\tau)-\lambda \bar{g}(\tau)-\left\langle\tau, \eta_{\mathrm{p}}^{0}\right\rangle\right\} \\
\text { subject to } \bar{g}(\tau)=0
\end{gathered}
$$

having the structure of a standard, constrained optimization problem as indicated in (2). Consequently, the analysis of Section 2 can be applied. The optimization conditions for (27) yield 
the equilibrium and the compatibility equations:

$$
\begin{gathered}
\nabla_{u} \Phi_{\mathrm{ep}}\left(C_{\eta} u\right)=C^{\prime} \tau(u)=f \\
C_{\eta} u=\nabla_{\tau} e^{\mathrm{c}}(\tau)+\lambda \nabla_{\tau} \bar{g}(\tau)+\eta_{\mathrm{p}}^{0} \\
\text { subject to } \bar{g}(\tau)=0
\end{gathered}
$$

In the following the standard iteration is described

1. Set the new external force value $f$.

(a) Initialization: $k=0$ and a tolerance $\delta$ for the external (equilibrium) loop is fixed.

(b) Initialization: $i=0$ and a tolerance $\varepsilon$ for the internal (constitutive) loop is fixed.

2. Predictor phase

(a) Starting point: $\lambda^{0}=0, \tau^{0}=\nabla_{\eta} e\left(\eta_{\text {tot }}-\eta_{\mathrm{p}}^{0}\right), u=u^{0}$

$\eta_{\text {tot }}=(C u, 0,0), u^{0}$ being the displacements (solution) at the past step.

(b) Evaluate the initial residuum $r=f-\nabla_{u} \Phi_{\mathrm{ep}}\left(C_{\eta} u^{0}\right)=f-C^{\prime} \nabla_{\eta} \Phi_{\mathrm{ep}}\left(C_{\eta} u^{0}\right)=f-C^{\prime} \tau^{0}$

(c) Evaluate the predictor $u=u^{0}+\delta u$ by solving the system $\left[C^{\prime} \nabla_{\eta, \eta}^{2} \Phi_{\mathrm{ep}}\left(C_{\eta} u^{0}\right) C\right] \delta u=r$.

The term $\nabla_{\eta, \eta}^{2} \Phi_{\mathrm{ep}}\left(C_{\eta} u^{0}\right)$ represents the tangent stiffness operator. It is evaluated from (28) assembling the local tangent operators $\nabla_{\eta} \tau\left(u^{0}\right)$ given by

$$
\begin{aligned}
\nabla_{\eta} \tau\left(u^{0}\right) & =G_{\tau}^{-1}-\frac{G_{\tau}^{-1} \nabla_{\tau} g\left(\tau^{0}\right) \otimes \nabla_{\tau} g\left(\tau^{0}\right) G_{\tau}^{-1}}{G_{\tau}^{-1} \nabla_{\tau} g\left(\tau^{0}\right) \cdot \nabla g\left(\tau^{0}\right)} \\
G_{\tau}\left(\tau^{0}\right) & =\nabla_{\tau, \tau}^{2} L\left(\tau^{0}, \lambda^{0}\right)=\nabla_{\tau, \tau^{c}}^{2} e^{\mathrm{c}}\left(\tau^{0}\right) \\
L\left(\tau^{0}, \lambda^{0}\right) & =e^{\mathrm{c}}\left(\tau^{0}\right)+\left\langle\tau^{0}, \eta_{\mathrm{p}}^{0}\right\rangle+\lambda^{0} \bar{g}\left(\tau^{0}, \lambda^{0}, c\right)-\left\langle\tau^{0}, C_{\eta} u^{0}\right\rangle
\end{aligned}
$$

Note that at the first iteration it is $\lambda=\lambda^{0}=0$ and $\bar{g}=0$. Equation (29) is the tangent stiffness operator at the point $u_{0}$.

3. Generic $k$ th iteration. Given $u^{k}$

(a) Evaluate the updated stress field $\tau^{k}$, the new Lagrangian multipliers $\lambda^{k}$ and the tangent stiffness operator solving the (local) system

$$
\left\{\begin{array}{l}
C_{\eta} u^{k}-\nabla_{\tau} e^{\mathrm{c}}\left(\tau^{k}\right)-\lambda^{k} \nabla_{\tau} \bar{g}\left(\tau^{k}\right)-\eta_{\mathrm{p}}^{0}=0 \\
-\bar{g}\left(\tau^{k}\right)=0
\end{array}\right.
$$

by means of Newton's iteration

$$
\begin{gathered}
{\left[\begin{array}{cc}
\nabla_{\tau, \tau}^{2} e^{\mathrm{c}}\left(\tau_{i}\right)+\lambda_{i} \nabla_{\tau, \tau}^{2} \bar{g} & \nabla_{\tau} \bar{g} \\
\nabla_{\tau}^{\mathrm{T}} \bar{g} & \nabla_{\lambda} \bar{g}
\end{array}\right]\left[\begin{array}{l}
\delta \tau_{i} \\
\delta \lambda_{i}
\end{array}\right]=\left[\begin{array}{c}
C_{\eta} u^{k}-\nabla_{\tau} e^{\mathrm{c}}\left(\tau_{i}\right)-\lambda_{i} \nabla_{\tau} \bar{g}-\eta_{\mathrm{p}}^{0} \\
-\bar{g}
\end{array}\right]} \\
\tau_{i+1}=\tau_{i}+\delta \tau_{i}, \quad \lambda_{i+1}=\lambda_{i}+\delta \lambda_{i}, \quad \bar{g}=\bar{g}\left(\tau_{i}, \lambda_{i}, c\right)
\end{gathered}
$$


where the gradients of the yield function $\nabla_{\tau} \bar{g}$ and $\nabla_{\lambda} \bar{g}$ are given by

$$
\nabla_{\tau} \bar{g}=\left\{\begin{array}{ll}
\nabla_{\tau} g & \text { if } g \geqslant-\lambda / c, \\
0 & \text { if } g<-\lambda / c,
\end{array} \quad \nabla_{\lambda} \bar{g}= \begin{cases}0 & \text { if } g \geqslant-\lambda / c \\
-1 / c & \text { if } g<-\lambda / c\end{cases}\right.
$$

The local tangent stiffness operator is evaluated by the inversion of system (31a) as

$$
\begin{aligned}
\nabla_{\eta} \tau\left(u^{k}\right) & =G_{\tau}^{-1}-\frac{G_{\tau}^{-1} \nabla_{\tau} g\left(\tau^{k}\right) \otimes \nabla_{\tau} g\left(\tau^{k}\right) G_{\tau}^{-1}}{G_{\tau}^{-1} \nabla_{\tau} g\left(\tau^{k}\right) \cdot \nabla g\left(\tau^{k}\right)} \\
G_{\tau}\left(\tau^{k}\right) & =\nabla_{\tau, \tau}^{2} L\left(\tau^{k}, \lambda^{k}\right)=\nabla_{\tau, \tau}^{2} e^{\mathrm{c}}\left(\tau^{k}\right)+\lambda^{k} \nabla_{\tau, \tau}^{2} \bar{g}\left(\tau^{k}, \lambda^{k}, c\right) \\
L\left(\tau^{k}, \lambda^{k}\right) & =e^{\mathrm{c}}\left(\tau^{k}\right)+\left\langle\tau^{k}, \eta_{\mathrm{p}}^{0}\right\rangle+\lambda \bar{g}\left(\tau^{k}, \lambda^{k}, c\right)-\left\langle\tau^{k}, C_{\eta} u^{k}\right\rangle
\end{aligned}
$$

In the case of multiple constraints equations (31a) become

$$
\left[\begin{array}{ccc}
E_{\mathrm{t}}^{-1}+\sum_{i=1, l} \lambda_{i} \nabla_{\tau, \tau}^{2} \bar{g}_{i} & \nabla_{\tau} \bar{g}_{a} & 0 \\
\nabla_{\tau}^{\mathrm{T}} \bar{g}_{a} & 0 & 0 \\
0 & 0 & -\frac{1}{c} I_{(p) \times(p)}
\end{array}\right]\left[\begin{array}{c}
\delta \tau \\
\delta \lambda_{a} \\
\delta \lambda_{\mathrm{p}}
\end{array}\right]=-\left[\begin{array}{c}
\nabla_{\tau} L\left(\tau_{i}, \lambda_{i}, c\right) \\
\bar{g}_{a}\left(\tau_{i}, \lambda_{i}, c\right) \\
-\lambda_{\mathrm{p}} / c
\end{array}\right]
$$

where the tangent elastic stiffness matrix has been indicated with $E_{\mathrm{t}}$, an index of the active constraints set, $a \in A=\left\{j \mid g_{j} \geqslant-\lambda_{j} / c\right\}$ with $a$ and the remaining inequality (inactive) constraints indices with $p$.

The local Newton's procedure is terminated when local convergence is reached

$$
\left\|\nabla_{\tau} L\left(\tau^{k}, \lambda^{k}\right)\right\|<\varepsilon
$$

(b) Evaluate the residual $r^{k}=f-C^{\prime} \tau^{k}$

(c) Update the displacements using Newton's step

$$
C^{\prime} \nabla_{\eta, \eta}^{2} \Phi_{\mathrm{ep}}\left(C_{\eta} u^{k}\right) C \delta u^{k}=r^{k} \longrightarrow u^{k+1}=u^{k}+\delta u^{k}
$$

(d) If the equilibrium residual norm meets the required tolerance $\delta$, the solution value of the displacements $u^{k+1}$ is reached, else repeat step 3 .

The positive constant $c$ utilized in the conversion of the inequality admissibility constraint into an equality one plays an important role in the definition of the active constraint set. Since for the inactive constraints we have $\bar{g}_{j}=-\lambda_{j} / c$, from the 3rd row of (34) it follows immediately that $\lambda_{j_{k+1}}=\lambda_{j_{k}}+c \bar{g}_{j}=0 \forall j \notin A$. Therefore, the iteration corresponds to set to zero the Lagrangian multipliers of the constraints that are not active, in the sense that $g_{j}<-\lambda_{j} / c$, and to treat the remaining active constraints in the same way as the equality constraints.

This observation shows one of the essential differences between the present approach, based on the substitution of the inequality constraint with an equality one and the traditional Lagrangian method (8), where the functional has to be optimized

$$
L^{+}(\tau, \lambda)=f(\tau)+\lambda g^{+}(\tau), \quad g^{+}(\tau)=\max [g(\tau), 0]
$$


The present approach leads also automatically to the determination of the correct Newton's iteration, with no need for physical, 'intuitive' interpretations.

\subsection{Multiplier method}

The Multiplier method described in Section 2 is obtained using the augmented Lagrangian regularization of the indicator function

$$
d^{\mathrm{c}}(\tau)=\operatorname{ind} K=\sup _{\lambda \in R}\left\{\lambda \bar{g}(\tau)+\frac{1}{2} c \bar{g}^{2}(\tau)\right\}
$$

so that the optimization problem becomes

$$
\begin{gathered}
\inf _{u}\left\{\Phi_{\mathrm{ep}}\left(C_{\eta} u\right)-\langle f, u\rangle\right\} \\
\Phi_{\mathrm{ep}}\left(C_{\eta} u\right)=\sup _{\tau} L_{c}(\tau, \lambda, c)=\sup _{\tau}\left\{\left\langle\tau, C_{\eta} u\right\rangle-e^{\mathrm{c}}(\tau)-\lambda \bar{g}(\tau)-\frac{1}{2} c \bar{g}^{2}(\tau)-\left\langle\tau, \eta_{\mathrm{p}}^{0}\right\rangle\right\}
\end{gathered}
$$

The iterative solution of the non-linear equilibrium equations is usually performed by means of classical Newton's or quasi Newton schemes, possibly associated with line search techniques. Then the Lagrangian multipliers are updated by means of formulas (10). The procedure is described in the sequel using a 1st order multiplier update.

1. Set the new external equivalent force value $f$.

(a) Initialization: $k=0$ and a tolerance $\gamma$ for the admissibility loop is fixed.

(b) Initialization: $j=0$ and a tolerance $\delta$ for the equilibrium loop is fixed.

(c) Initialization: $i=0$ and a tolerance $\varepsilon$ for the constitutive loop is fixed.

2. Predictor phase.

(a) Starting point: $\lambda^{0}=0, \tau^{0}=\nabla_{\eta} e\left(\eta_{\mathrm{tot}}-\eta_{\mathrm{p}}^{0}\right), u=u^{0}, \eta_{\mathrm{tot}}=(C u, 0,0), u^{0}$ being the displacements (solution) at the past iteration.

First update of $\lambda: \lambda^{1}=\lambda^{0}+c^{0} g\left(\tau^{0}, c^{0}\right)=0$ (since at the end of the previous step the constraints were satisfied within the required tolerance).

Set $c^{1}=c^{0}$.

(b) Minimize (w.r.t. $u$ ) the functional $L_{c}\left(\tau, \lambda^{1}, c^{1}\right)=\left\langle\tau, C_{\eta} u\right\rangle-e^{\mathrm{c}}(\tau)-\lambda^{1} \bar{g}(\tau)-\frac{1}{2} c^{1} \bar{g}^{2}(\tau)-$ $\left\langle\tau, \eta_{\mathrm{p}}^{0}\right\rangle$

This can be done either using a search method or a Newton's iterations. In the latter case, the following steps are needed:

i. Evaluate the initial residuum $r=f-C^{\prime} \nabla_{\eta} \Phi_{\mathrm{ep}}\left(C_{\eta} u^{0}\right)=f-C^{\prime} \tau^{0}$.

ii. Evaluate the predictor $u=u^{0}+\delta u$ solving the system $\left[C^{\prime} \nabla_{\eta, \eta}^{2} \Phi_{\mathrm{ep}}\left(C_{\eta} u^{0}\right) C\right] \delta u=r$. In this case (compare with (29)) the local tangent stiffness operator $\nabla_{\eta} \tau\left(u^{0}\right)$ is equal to

$$
\begin{aligned}
\nabla_{\eta} \tau\left(u^{0}\right) & =\left[\nabla_{\tau, \tau}^{2} L_{c}\left(\tau^{0}, \lambda^{1}, c^{1}\right)\right]^{-1} \\
& =\left[\nabla_{\tau, \tau}^{2} e^{\mathrm{c}}\left(\tau^{0}\right)+c^{1} \bar{g}\left(\tau^{0}\right) \nabla_{\tau, \tau}^{2} \bar{g}\left(\tau^{0}\right)+c^{1} \nabla_{\tau} \bar{g}\left(\tau^{0}\right) \otimes \nabla_{\tau} \bar{g}\left(\tau^{0}\right)\right]^{-1}
\end{aligned}
$$


iii. Iterate until equilibrium is reached: $r_{j}^{1}=f-C^{\prime} \tau_{j} \leqslant \delta$. Then set $u^{1}=u$.

Each iteration requires the evaluation of the updated internal forces $\tau_{j}$ and of the tangent stiffness operator, obtained solving the system:

$$
\begin{aligned}
& {\left[\nabla_{\tau, \tau}^{2} e^{\mathrm{c}}\left(\tau_{i}\right)+c^{1} \bar{g}\left(\tau_{i}\right) \nabla_{\tau, \tau}^{2} \bar{g}\left(\tau_{i}\right)+c^{1} \nabla_{\tau} \bar{g}\left(\tau_{i}\right) \otimes \nabla_{\tau} \bar{g}\left(\tau_{i}\right)\right]\left[\delta \tau_{i}\right]} \\
& \quad=\left[\eta_{\mathrm{tot}}-\nabla_{\tau} e^{\mathrm{c}}\left(\tau_{i}\right)-c^{1} \bar{g}\left(\tau_{i}\right) \nabla_{\tau} \bar{g}\left(\tau_{i}\right)-\eta_{\mathrm{p}}^{0}\right]
\end{aligned}
$$

Note that, since $\lambda^{1}=0, \bar{g} \equiv g^{+}$.

3. Generic iteration. Start with estimates $u^{k}, \lambda^{k}$;

(a) Update $\lambda$, update $c$.

$$
\begin{array}{ll}
1 \text { st order multiplier update (10a): } & \lambda^{k+1}=\lambda^{k}+c^{k} \bar{g}\left(\tau^{k}\right) \\
\text { Penalty parameter update: } & c_{k} \rightarrow c_{k+1}
\end{array}
$$

(b) Minimize the functional $\Phi_{\mathrm{ep}}\left(C_{\eta} u\right)-\langle f, u\rangle$ keeping the Lagrangian multipliers fixed. Each iteration requires the evaluation of the updated internal forces and of the tangent stiffness operator, obtained solving the system

$$
\eta_{\text {tot }}-\nabla_{\tau} e^{\mathrm{c}}\left(\tau_{i}\right)-\left(\lambda^{k+1}+c^{k+1} \bar{g}\left(\tau_{i}\right)\right) \nabla_{\tau} \bar{g}\left(\tau_{i}\right)-\eta_{\mathrm{p}}^{0}=0
$$

eventually by means of a Newton's procedure

$$
\begin{aligned}
\tau_{i+1}= & \tau_{i}+\left[\nabla_{\tau, \tau}^{2} e^{\mathrm{c}}\left(\tau_{i}\right)+\left(\lambda^{k+1}+c^{k+1} \bar{g}\left(\tau_{i}\right)\right) \nabla_{\tau, \tau}^{2} \bar{g}\left(\tau_{i}\right)+c^{k+1} \nabla_{\tau} \bar{g}\left(\tau_{i}\right) \otimes \nabla_{\tau} \bar{g}\left(\tau_{i}\right)\right]^{-1} \\
& \cdot\left[\eta_{\mathrm{tot}}-\nabla_{\tau} e^{\mathrm{c}}\left(\tau_{i}\right)-\left(\lambda^{k+1}+c^{k+1} \bar{g}\left(\tau_{i}\right)\right) \nabla_{\tau} \bar{g}\left(\tau_{i}\right)-\eta_{\mathrm{p}}^{0}\right]
\end{aligned}
$$

The local Newton's procedure is terminated when local convergence is reached

$$
\left\|\nabla_{\tau} L_{c}\left(\tau^{k+1}, \lambda^{k+1}, c^{k+1}\right)\right\|<\varepsilon
$$

In this case the algorithmic tangent stiffness operator is given by

$$
\nabla_{\eta} \tau\left(u^{k}\right)=\left[\begin{array}{c}
\nabla_{\tau, \tau}^{2} e^{\mathrm{c}}\left(\tau^{k+1}\right)+\left(\lambda^{k+1}+c^{k+1} \bar{g}\left(\tau^{k+1}\right)\right) \nabla_{\tau, \tau}^{2} \bar{g}\left(\tau^{k+1}\right) \\
+c^{k+1} \nabla_{\tau} \bar{g}\left(\tau^{k+1}\right) \otimes \nabla_{\tau} \bar{g}\left(\tau^{k+1}\right)
\end{array}\right]^{-1}
$$

(c) When the equilibrium residual norm meets the required tolerance

$$
\left\|\left[\nabla_{u} \Omega_{R}\right]\right\|<\delta
$$

the new value of the displacements $u^{k+1}$ is reached.

4. Check the admissibility convergence. If the total number of control points is $n_{\mathrm{cp}}$, using condition (12), the convergence test is

$$
\max _{n=1, n_{\mathrm{cp}}}\left|\bar{g}\left(\tau^{k+1}\right)\right|_{n}<\gamma
$$

If it is not satisfied go to 3 . 
In the Multiplier algorithm the converged stresses at each equilibrium iteration (step 3(b) in the above scheme) do not satisfy the admissibility conditions, since they are obtained from (41) keeping the Lagrangian multipliers fixed. In this sense the constraint is 'relaxed' at each iteration. Furthermore, since the Lagrangian multipliers are fixed, the inelastic deformation rates keep a constant magnitude during the equilibrium iteration, still changing their direction, that is orthogonal to the gradient of the plastic potential $\nabla_{\tau} \bar{g}\left(\tau_{i}\right)$.

Each equilibrium iteration requires in both methods the evaluation of the updated internal forces and of the tangent stiffness operator, obtained solving the local systems of stationarity conditions (see (30) and (40)), eventually by means of a Newton's procedure.

In the case of damaging models, additional constraints may appear in this local process of estimation of the elastic-plastic tangent operator $\Phi_{\mathrm{ep}}\left(C_{\eta} u\right)$. In fact it is well known that in the case of isotropic damage the elastic damage variable $\omega_{\mathrm{e}}$ has to satisfy the two-sided inequality $-1 \leqslant \omega_{\mathrm{e}} \leqslant 0$ that leads to the inequality constraints $h_{1}=-\left(1+\omega_{\mathrm{e}}\right) \leqslant 0, h_{2}=\omega_{\mathrm{e}} \leqslant 0$, converted, similarly to the constraint $g(\tau) \leqslant 0$, into the equality ones by means of the following functions:

$$
\bar{h}_{1}=\max \left(h_{1},-\beta_{1} / c_{1}\right), \quad \bar{h}_{2}=\max \left(h_{2},-\beta_{2} / c_{2}\right), \quad \beta_{1}, \beta_{2} \in R, \quad c_{1}, c_{2} \in R^{+}
$$

Because of the presence of these constraints and of the non-convex nature of the internal energy, the elastic constitutive equations cannot be inverted in close form, so a numerical procedure is adopted. The elastic-plastic tangent operator is evaluated through the following expression, related to the case of the Multiplier method:

$$
\Phi_{\mathrm{ep}}\left(C_{\eta} u\right)=\sup _{\tau} \inf _{\lambda} \inf _{\eta_{\mathrm{e}}} \operatorname{stat}_{\left(\beta_{1}, \beta_{2}\right)}\left\{\begin{array}{c}
\left\langle\tau, C_{\eta} u\right\rangle-\left[\left\langle\tau, \eta_{\mathrm{e}}\right\rangle-e\left(\eta_{\mathrm{e}}\right)-\beta_{1} \bar{h}_{1}-\beta_{2} \bar{h}_{2}\right] \\
-\lambda \bar{g}(\tau)-\frac{1}{2} c \bar{g}^{2}(\tau)-\left\langle\tau, \eta_{\mathrm{p}}^{0}\right\rangle
\end{array}\right\}
$$

whose stationarity equations are

$$
\left\{\begin{array}{l}
C_{\eta} u-\eta_{\mathrm{e}}-(\lambda+c \bar{g}(\tau)) \nabla_{\tau} \bar{g}(\tau)-\eta_{\mathrm{p}}^{0}=0 \\
-\tau+\nabla_{\eta_{\mathrm{e}}} e\left(\eta_{\mathrm{e}}\right)+\beta_{1} \nabla_{\eta_{\mathrm{e}}} \bar{h}_{1}+\beta_{2} \nabla_{\eta_{\mathrm{e}}} \bar{h}_{2}=0 \\
\bar{h}_{1}=0 \\
\bar{h}_{2}=0
\end{array}\right.
$$

A Newton's scheme is adopted for the solution of the non-linear system given by the $1 \mathrm{st}$, the 3 rd and the 4 th of (44) in the unknowns $\left(\tau, \beta_{1}, \beta_{2}\right)$, after a condensation w.r.t the variables $\eta_{\mathrm{e}}$. The update of the kinematic variables is given by

$$
\begin{aligned}
\eta_{\mathrm{e}_{i+1}}=\eta_{\mathrm{e}_{i}}+\Delta \eta_{\mathrm{e}_{i}}, \quad \Delta \eta_{\mathrm{e}_{i}} & =-\left[\nabla_{\eta_{\mathrm{e}} \eta_{\mathrm{e}}}^{2} e\left(\eta_{\mathrm{e}}\right)\right]_{i}^{-1}\left[R_{\eta_{\mathrm{e}}}-\Delta \tau+\Delta \beta_{1} \nabla_{\eta_{\mathrm{e}}} \bar{h}_{1}+\Delta \beta_{2} \nabla_{\eta_{\mathrm{e}}} \bar{h}_{2}\right]_{i} \\
R_{\eta_{\mathrm{e}}} & =-\tau+\nabla_{\eta_{\mathrm{e}}} e\left(\eta_{\mathrm{e}}\right)+\beta_{1} \nabla_{\eta_{\mathrm{e}}} \bar{h}_{1}+\beta_{2} \nabla_{\eta_{\mathrm{e}}} \bar{h}_{2}
\end{aligned}
$$

The use of the iterative update (45) for the elastic internal variables also avoids problems in points of non-definiteness of the internal energy functional, which occurs for instance in relation to the form introduced by the authors in Reference [15] and that will be used in the numerical applications of the subsequent section. An identical procedure can be developed in the case of the Lagrangian method. 


\section{NUMERICAL APPLICATIONS}

The algorithms described in Section 3 will now be applied to specific elastic-plastic problems characterized by strong coupling between plasticity, hardening and damage.

A material model is employed for which the reversible behaviour of the material is ruled by the following elastic potential:

$$
e\left(\eta_{\mathrm{e}}\right)=\frac{1}{2} E \varepsilon_{\mathrm{e}} \cdot \varepsilon_{\mathrm{e}}\left(1+\omega_{\mathrm{e}}\right)^{n}+\frac{1}{2} H \alpha_{\mathrm{e}} \cdot \alpha_{\mathrm{e}}\left(1+\omega_{\mathrm{e}}\right)^{n}
$$

$E$ and $H$ being, respectively, the tangent elastic and hardening operators, $\varepsilon_{\mathrm{e}}$ the elastic strain, $\alpha_{\mathrm{e}}$ the elastic hardening variable and $\omega_{\mathrm{e}}$ the internal scalar damage variable. The exponent $n$ rules the isotropic damage law.

The irreversible behaviour is ruled by the yield function $g$, which, as early specified, defines the conjugated dissipation functional. A different form of the elastic domain will be considered, depending on the numerical test performed. However, in each case, the following general expression of the yield function has been considered:

$$
g(\sigma, \chi, \zeta)=f\left(I_{1}, J_{2}, J_{3}, w\right)
$$

It depends on the invariants of the stress tensor $\sigma$ and of the deviator of stresses $\sigma^{\prime}$

$$
\begin{aligned}
& I_{1}=\operatorname{tr}(\sigma) \\
& J_{2}=\frac{1}{2} \sigma^{\prime} \cdot \sigma^{\prime}, \quad \sigma_{i j}^{\prime}=\sigma_{i j}-\frac{1}{3} \sigma_{q q} \delta_{i j} \\
& J_{3}=\operatorname{det} \sigma^{\prime}
\end{aligned}
$$

and on the limit elastic resistance $w=k_{0}+\chi-k \zeta, k_{0}$ and $k$ being material constitutive parameters.

The algorithms presented in Section 3 have been implemented into a FORTRAN code, namely multi surfaces damage model (MSDM), implemented on the skeleton of the FEAP code [16]. A 1st order update scheme has been employed in the multiplier method throughout the following applications.

\subsection{An elastic-plastic model based on Ottosen criterion}

In this section an elastic-plastic problem with an elastic domain that presents regions of sharp curvature is studied. Specifically, the behaviour of the numerical algorithms proposed in Section 3 has been tested with a generalized Ottosen criterion, including isotropic hardening and damage [17].

The yield function has the form

$$
\begin{gathered}
g=\frac{a}{y_{\mathrm{c}}+\chi-k \zeta} J_{2}+\lambda(\cos 3 \theta(\sigma)) \sqrt{J_{2}}+b I_{1}-\left(y_{\mathrm{c}}+\chi-k \zeta\right) \\
a, b, y_{\mathrm{c}}, k \in R^{+}, \quad \cos 3 \theta(\sigma)=\frac{3}{2} \frac{3 \sqrt{J_{3}}}{J_{2} \sqrt{J_{2}}} \\
\lambda(\cos 3 \theta(\sigma))= \begin{cases}k_{1} \cos \left[\frac{1}{3} \arccos \left(k_{2} \cos 3 \theta(\sigma)\right)\right], & \cos 3 \theta \geqslant 0, \quad k_{1}>0 \\
k_{1} \cos \left[\pi-\frac{1}{3} \arccos \left(-k_{2} \cos 3 \theta(\sigma)\right)\right], & \cos 3 \theta<0, \quad 0 \leqslant k_{2}<1\end{cases}
\end{gathered}
$$


The parameters $a$ and $b$ rule the shape of the meridian sections, and $k_{1}$ and $k_{2}$ control, respectively, the size and the shape of the deviatoric section, varying from circular $\left(k_{2}=0\right)$ to triangular $\left(k_{2}=1\right)$. In (47) $y_{\mathrm{c}}$ is the limit elastic resistance in uniaxial compression, while the corresponding resistance in tension will be denoted by $y_{t}$.

A clear representation of the surface and of the stress paths is obtained using the HaighWestergaard co-ordinates: the hydrostatic component $\xi=I_{1} / \sqrt{3}$, the deviatoric component $r=\sqrt{2 J_{2}}$ and the Lode angle $\theta$. The following expression is obtained:

$$
g(\xi, r, \theta, \chi, \zeta)=a \frac{r^{2}}{2\left(y_{\mathrm{c}}+\chi-\zeta\right)}+\lambda(\cos 3 \theta) \frac{r}{\sqrt{2}}+b \sqrt{3} \xi-\left(y_{\mathrm{c}}+\chi-k \zeta\right)
$$

The constitutive parameters have been deduced by means of a data fitting procedure, using two sets of data.

From the experimental data of Van Mier [18] the following values have been obtained: $y_{\mathrm{c}}=16.40 \mathrm{~N} / \mathrm{mm}^{2}, y_{\mathrm{t}}=2.95 \mathrm{~N} / \mathrm{mm}^{2}, k_{1}=7.044261, k_{2}=0.8999994, a=0.00023861$, $b=1.53818$, initial elastic modulus $E=27000 \mathrm{~N} / \mathrm{mm}^{2}$, Poisson ratio $v=0.3$. These have been used in the applications in perfect or hardening plasticity.

A coupled damage model has been tuned on the experimental data of Kupfer [19], obtaining $y_{\mathrm{c}}=7.29 \mathrm{~N} / \mathrm{mm}^{2}, y_{\mathrm{t}}=1.29 \mathrm{~N} / \mathrm{mm}^{2}, k_{1}=6.9377, k_{2}=0.9043, a=0.471514, b=1.65913$, initial elastic modulus $E=20000 \mathrm{~N} / \mathrm{mm}^{2}$, Poisson ratio $v=0.2$, damage parameters $k=30$, $n=2$.

Both sets of parameters are such that the surface presents a nearly triangular deviatoric section for positive values of the hydrostatic stress. Several different values for the hardening modulus have been adopted, as will be specified.

4.1.1. Gauss point convergence. A first set of analyses concerns the convergence properties of the (local) compatibility system (30) obtained in the implementation of the Lagrangian method described in Section 3.1. This is the convergence check that is usually addressed in numerical studies $[4,5,8]$. It is stressed once more that algorithm (31a) stemming out from (30) differs from the standard Lagrangian method for the introduction of the equivalent equality constraint $\bar{g}$ that requires the introduction of a constant real augmentation parameter $c$.

The local convergence properties (at a single Gauss point) have been investigated in a deformation driven process. Starting from an initial elastic state located on the limit surface, a total strain increment $\left(\varepsilon_{1}, \varepsilon_{2}, \varepsilon_{3}\right)$ has been assigned and it has been characterized by the initial elastic trial stress state. Because the initial state is virgin, the accumulated plastic strains $\varepsilon_{\mathrm{p}}^{0}$ are null and the assigned total strain increment coincides with an elastic increment. It is such that the corresponding elastic predictor satisfies prescribed values of $(\xi, r, \theta)$. Specifically, the stress components have been evaluated solving numerically the non-linear system

$$
\left(\sigma_{1}, \sigma_{2}, \sigma_{3}\right):\left\{\begin{array}{l}
r_{\text {trial }}=\sqrt{2 J_{2}\left(\sigma_{1}, \sigma_{2}, \sigma_{3}\right)} \\
\xi_{\text {trial }}=\frac{I_{1}\left(\sigma_{1}, \sigma_{2}, \sigma_{3}\right)}{\sqrt{3}} \\
\theta_{\text {trial }}=\frac{1}{3} \cos ^{-1}\left[\frac{3 \sqrt{3}}{2} \frac{J_{3}\left(\sigma_{1}, \sigma_{2}, \sigma_{3}\right)}{\sqrt{J_{2}^{3}\left(\sigma_{1}, \sigma_{2}, \sigma_{3}\right)}}\right]
\end{array}\right.
$$


A wide range of trial states has been investigated, considering two different trial values for the hydrostatic stress $\xi_{\text {trial }}$, one greater than zero denoted by $T 1$, the other smaller than zero denoted by $T 3$. For each value of $\xi_{\text {trial }}$, several values of the Lode angle in the range $0^{\circ} \leqslant \theta \leqslant 60^{\circ}$ and of the radial distance $r$ have been considered. The absolute values of the trial $\xi$ are taken equal to the value of the radial measure $r_{0}$ of the Ottosen domain for $\xi=0$ and $\theta=30^{\circ}$, so that $T 1=-r_{0}, T 3=r_{0}$ (Figure 5(a)). From the limit elastic value $r_{\mathrm{el}}$, the trial radius is iteratively incremented for each angle $\theta$ of a constant $\Delta r$ (see Figures 5(b) and (c)), such as

$$
\begin{aligned}
& \left\{\begin{array}{ll}
r_{\text {trial }}=r_{\mathrm{el}}+n \Delta r, & n=1, \ldots, 50 \\
r_{\mathrm{el}} \leqslant r_{\text {trial }} \leqslant r_{\mathrm{el}}+50 \Delta r, & \Delta r=0.2 \text { if } \xi=T 1 \\
r_{\mathrm{el}} \leqslant r_{\text {trial }} \leqslant r_{\mathrm{el}}+50 \Delta r, & \Delta r=0.1 \text { if } \xi=T 3
\end{array}\right. \text { Perfect plasticity } \\
& \begin{cases}r_{\text {trial }}=r_{\mathrm{el}}+n \Delta r, & n=1, \ldots, 60 \\
r_{\mathrm{el}} \leqslant r_{\text {trial }} \leqslant r_{\mathrm{el}}+60 \Delta r, & \Delta r=0.1, \quad \xi=T 1, \quad \xi=T 3 \quad \text { Damaging plasticity }\end{cases}
\end{aligned}
$$

For the Lode angle a step $\Delta \theta=1^{\circ}$ has been adopted, so that the simulations have been performed over a grid of 3000 trial states in the case of perfect plasticity and over a grid of 3600 trial states in the presence of damage.

The number of Newton's iterations needed for convergence to the solution of the constitutive equations are reported in Figure 6 in the case of perfect plasticity and in Figure 7 for damaging plasticity, for both the trial values of $\xi$.

It is obtained that, for any trial state and for all the Lode angles, even with rather large increments of the deviatoric component, convergence is reached in a very limited number of steps. Apparently the loss of convergence claimed by other authors for angles close to $0^{\circ}$ and $60^{\circ}$ is not observed in this case, even for a positive trace, in which case the criterion shows a very strong triangular-like deviatoric section (see Figure 5(b)). This is possibly due to the regularization adopted for the dissipation functional, that avoids discontinuities around zero [15].

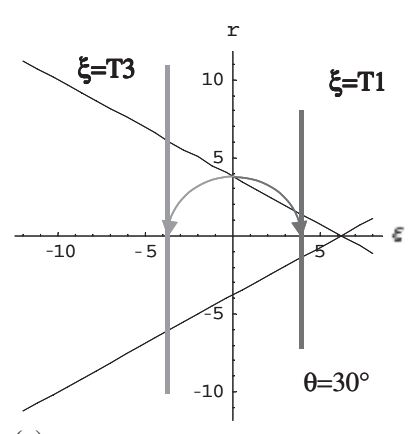

(a)

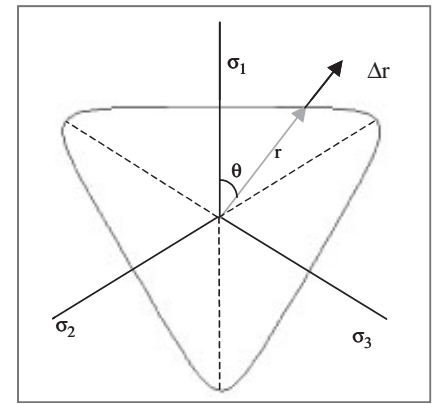

(b)

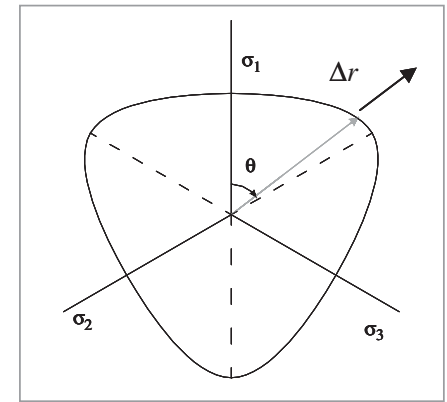

(c)

Figure 5. Definition of the trial state maps: (a) trial values of the hydrostatic components on the meridian plane for $\theta=30^{\circ}$; (b) deviatoric section in the tension region, $\xi=T 1$; and (c) deviatoric section in the compression region, $\xi=T 3$. 

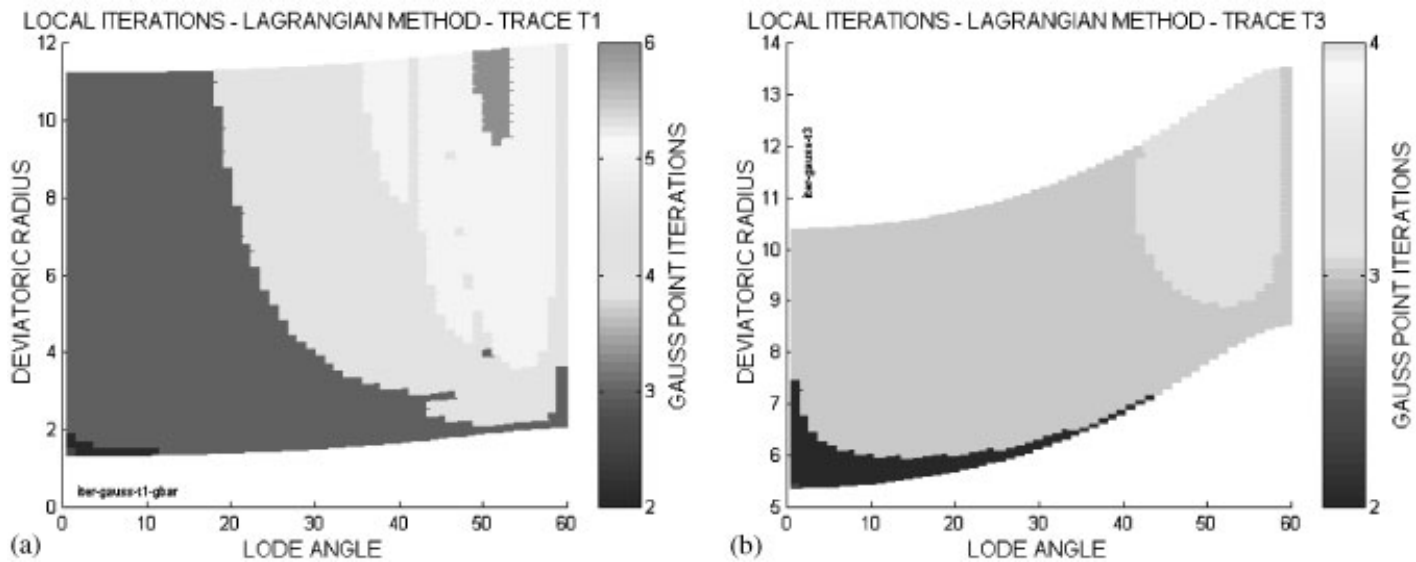

Figure 6. Number of Newton's iterations for the convergence at Gauss point. Lagrangian method. Perfect plasticity: (a) $\xi=T 1$; and (b) $\xi=T 3$.
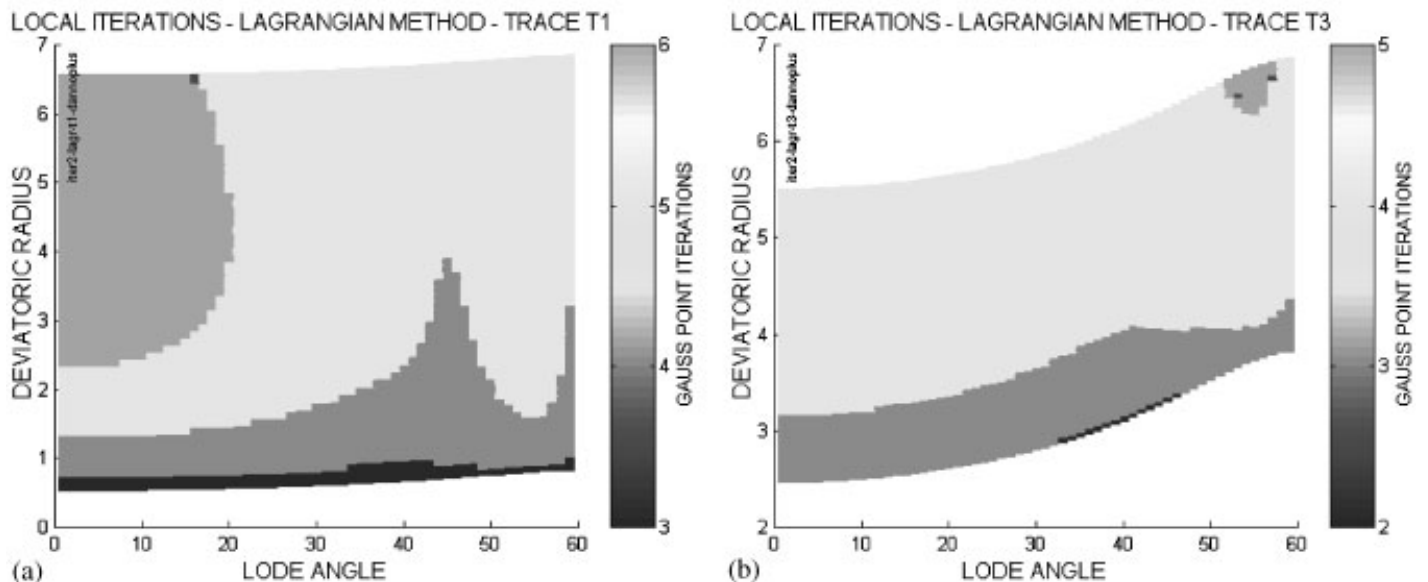

Figure 7. Number of Newton's iterations for the convergence at Gauss point. Lagrangian method. Damaging plasticity: (a) $\xi=T 1$; and (b) $\xi=T 3$.

Since the tests have been carried out at imposed deformation, it is important to evaluate at what point of the yield surface the stresses converge.

The contour level maps of Figure 8 refer to the case $\xi=T 1$ for perfect plasticity. Plots 8 (a) and 8(b), shows, respectively, the contour level of the ratio $r_{\text {conv }} / r_{\mathrm{el}}$ and of the Lode angle at convergence $\theta_{\text {conv }}, r_{\text {conv }}$ being the value assumed by the deviatoric component at the solution, and $r_{\mathrm{el}}$ the radius of the elastic domain for the assigned $\theta$. Both plots suggest that the solution stress state migrates towards the region with higher curvature, especially for trial states having a Lode angle greater than $30^{\circ}$. Indeed a vertical level in Figure 8(b) indicates that stresses return to the limit surface at the same angle of the trial initial state. Simultaneously, from 

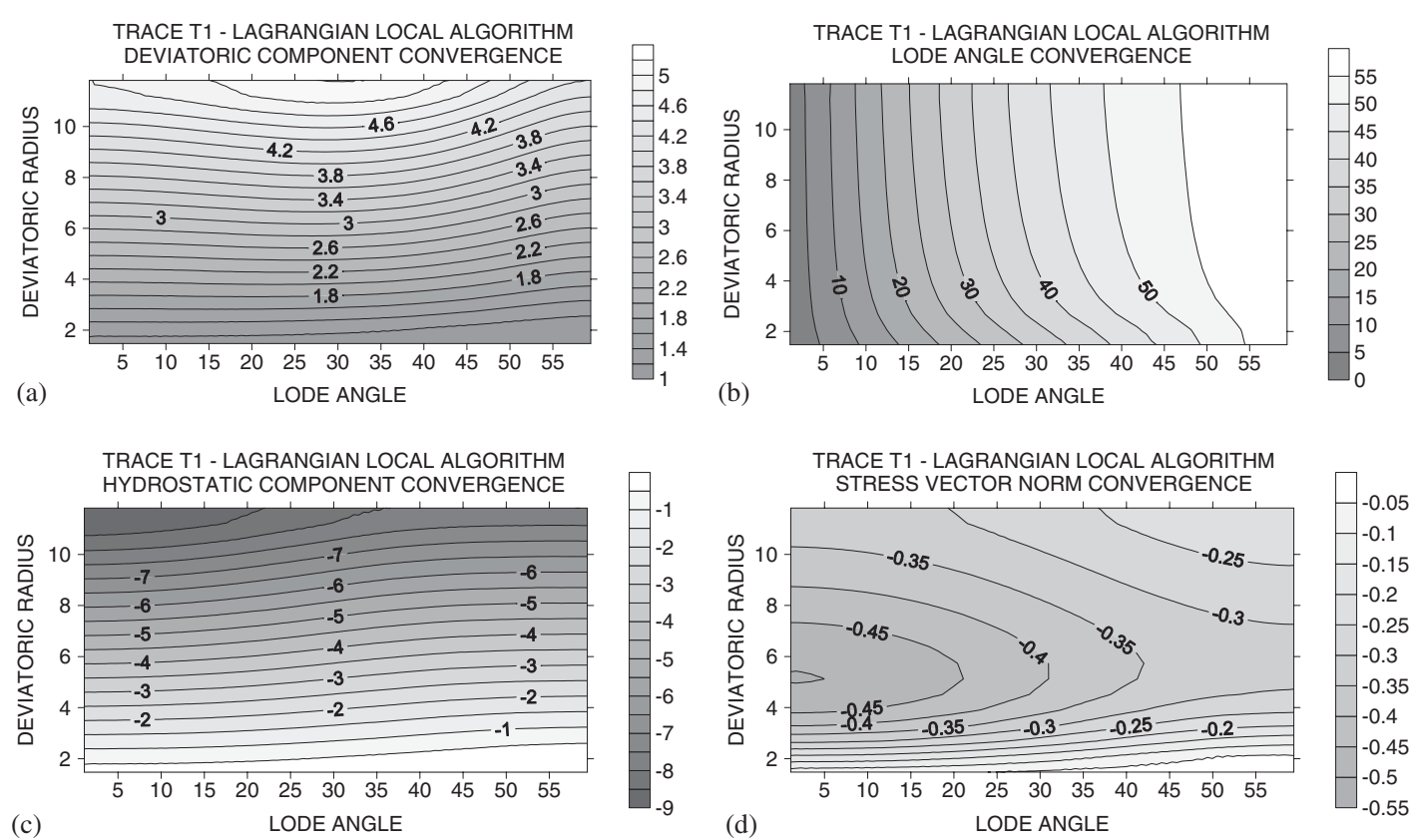

Figure 8. Iso-value maps of stress convergence. $\xi=T 1$. Lagrangian method. Perfect plasticity: (a) deviatoric component; (b) Lode angle; (c) hydrostatic component; and (d) stress vector norm.

plot $8(\mathrm{c})$, where the difference $\xi_{\text {conv }}-\xi_{\text {trial }}$ is reported, $\xi_{\text {conv }}$ being the hydrostatic stresses at solution, it is observed that the solution point moves in the stress space towards stress vectors with a smaller hydrostatic component, going faraway from the apex. In picture 8(d) the contour levels of the ratio $\left(\|\sigma\|_{\text {conv }}-\|\sigma\|_{\mathrm{el}}\right) /\|\sigma\|_{\mathrm{el}}$ are represented, $\|\sigma\|=\sqrt{r^{2}(\theta)+\xi^{2}(\theta)}$ being the amplitude of the stress vector. Similar considerations can be made analysing the contour level maps of Figure 9 in the case $\xi=T 1$ for damaging plasticity.

In the case $\xi=T 3$, corresponding to a region with compressive hydrostatic component, the curvature of the meridian section is less severe than in the previous situations, but a similar trend for the convergence properties is revealed. It is important to observe that in this case the stress returns to points not far from the initial trial state. The results are summarized in Figure 10 for perfect plasticity and in Figure 11 for damaging plasticity.

4.1.2. Equilibrium convergence. The results of the previous section have shown that, even if convergence at Gauss point appears to be always reached, the converged stresses can be quite different from the trial ones. Therefore, the question arises about convergence of the equilibrium problem. Indeed, the numerical calculations often show lack of convergence in the fulfilment of the non-linear equilibrium equations. Since the equilibrium problem involves the optimization of functional (25) Lagrangian method or Multiplier method can be used.

In order to analyse this point, a very simple test problem has been studied, namely nodal forces have been assigned in such a way as to generate a homogeneous state of stress, and hardening has been added to the elastic-plastic behaviour of the material. Starting from a limit 


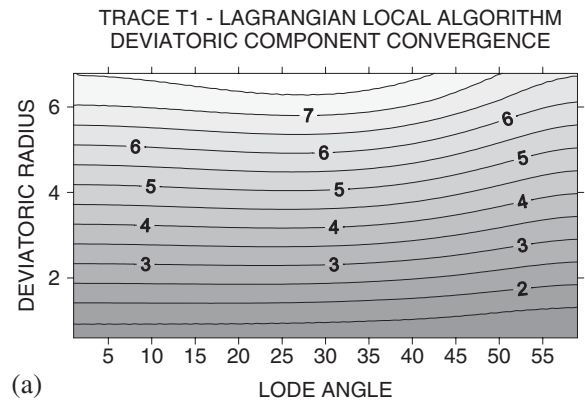

TRACE T1 - LAGRANGIAN LOCAL ALGORITHM HYDROSTATIC COMPONENT CONVERGENCE

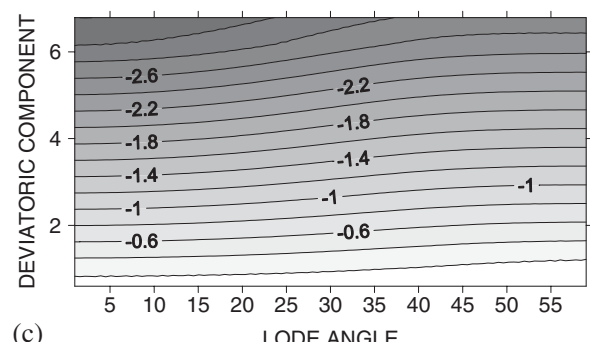

(c)
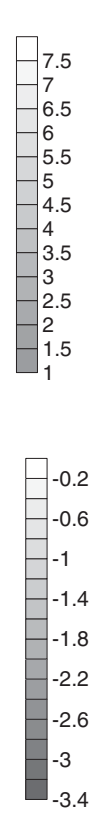

TRACE T1 - LAGRANGIAN LOCALALGORITHM LODE ANGLE CONVERGENCE

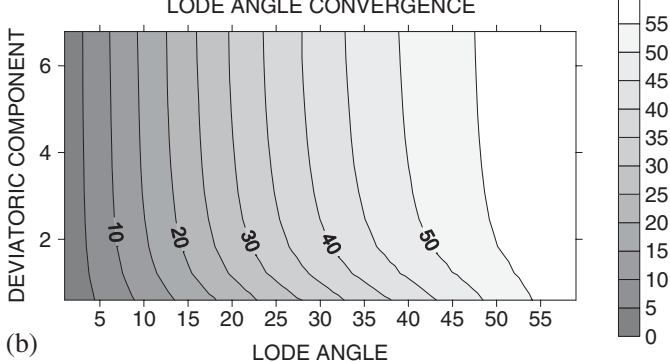

TRACE T1 - LAGRANGIAN LOCAL ALGORITHM STRESS VECTOR NORM CONVERGENCE

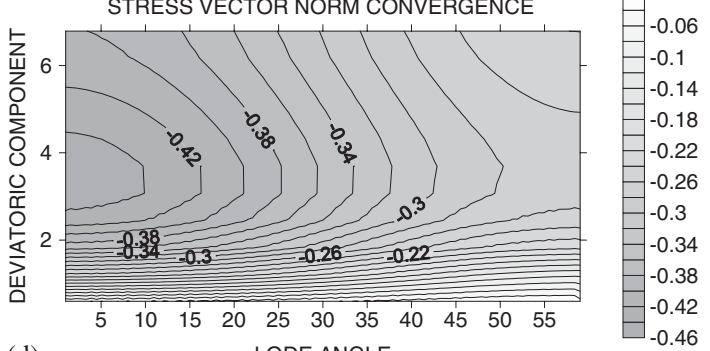

(d)

Figure 9. Iso-value maps of stress convergence. $\xi=T 1$. Lagrangian method. Damaging plasticity: (a) deviatoric component; (b) Lode angle; (c) hydrostatic component; and (d) stress vector norm.
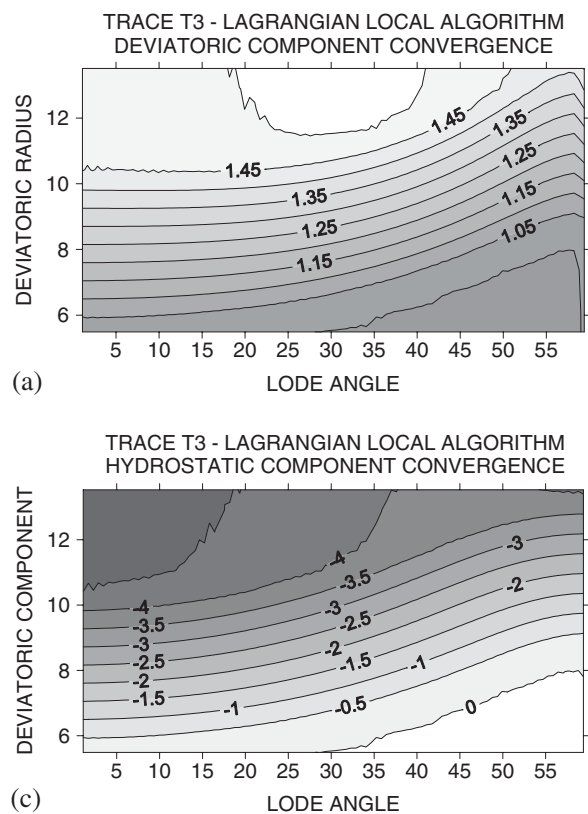
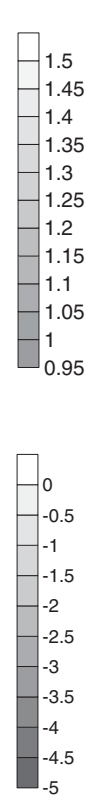

TRACE T3 - LAGRANGIAN LOCAL ALGORITHM

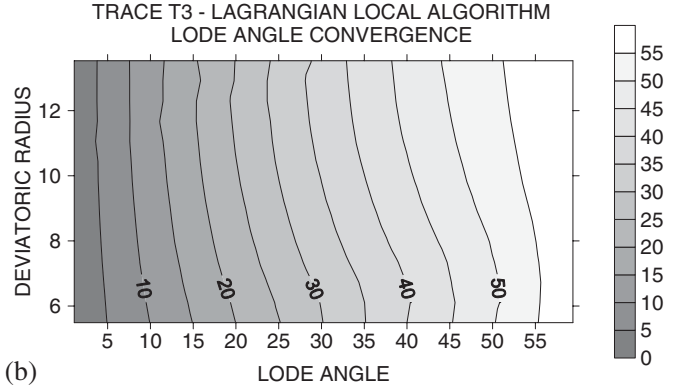

(b)

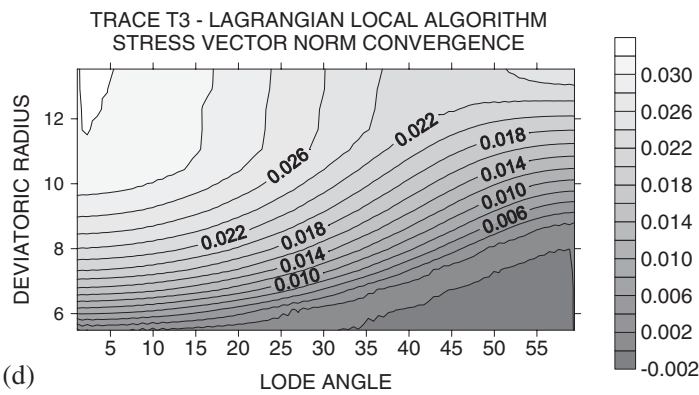

Figure 10. Iso-value maps of stress convergence. $\xi=T 3$. Lagrangian method. Perfect plasticity: (a) deviatoric component; (b) Lode angle; (c) hydrostatic component; and (d) stress vector norm. 


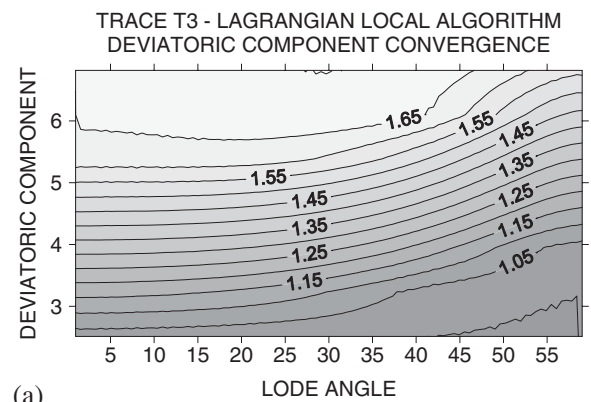

(a)

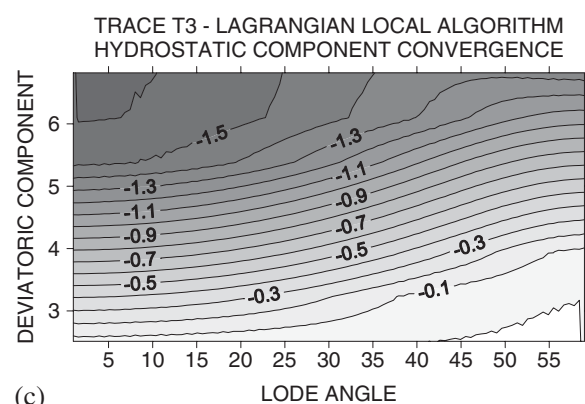

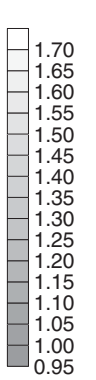

넘

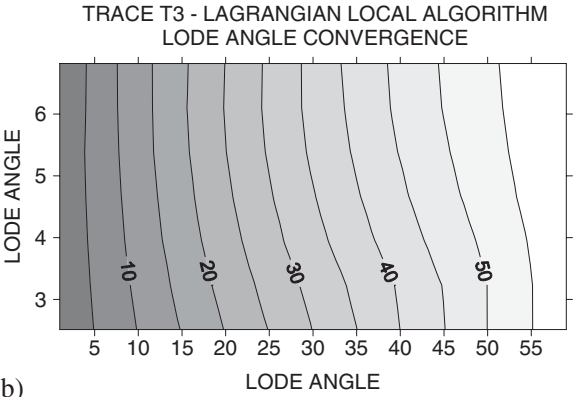

(b)
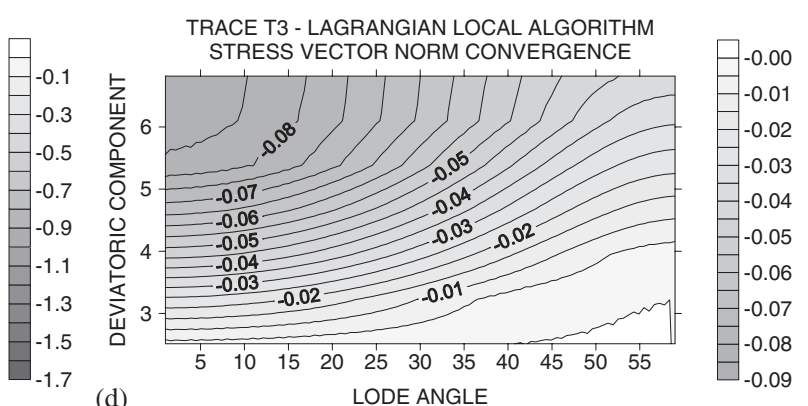

Figure 11. Iso-value maps of stress convergence. $\xi=T 3$. Lagrangian method. Damaging plasticity: (a) deviatoric component; (b) Lode angle; (c) hydrostatic component; and (d) stress vector norm.
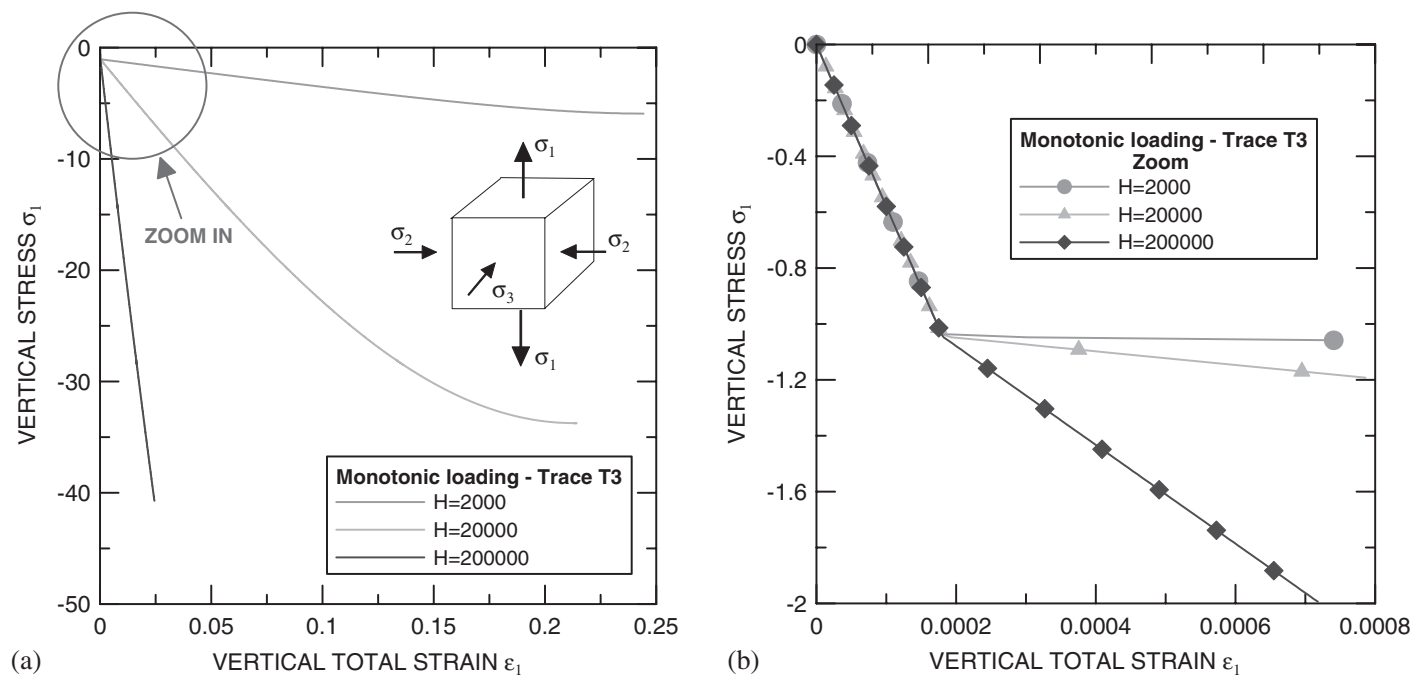

Figure 12. Monotonic loading. Pre-peak region. $\xi=T 3$. Damaging plasticity.

elastic state, the increments of nodal forces have been assigned such that the corresponding elastic strains coincide with the increments used in the local analysis at the Gauss point level of the previous section. In this way, all the elastic predictors constitute a subset of 

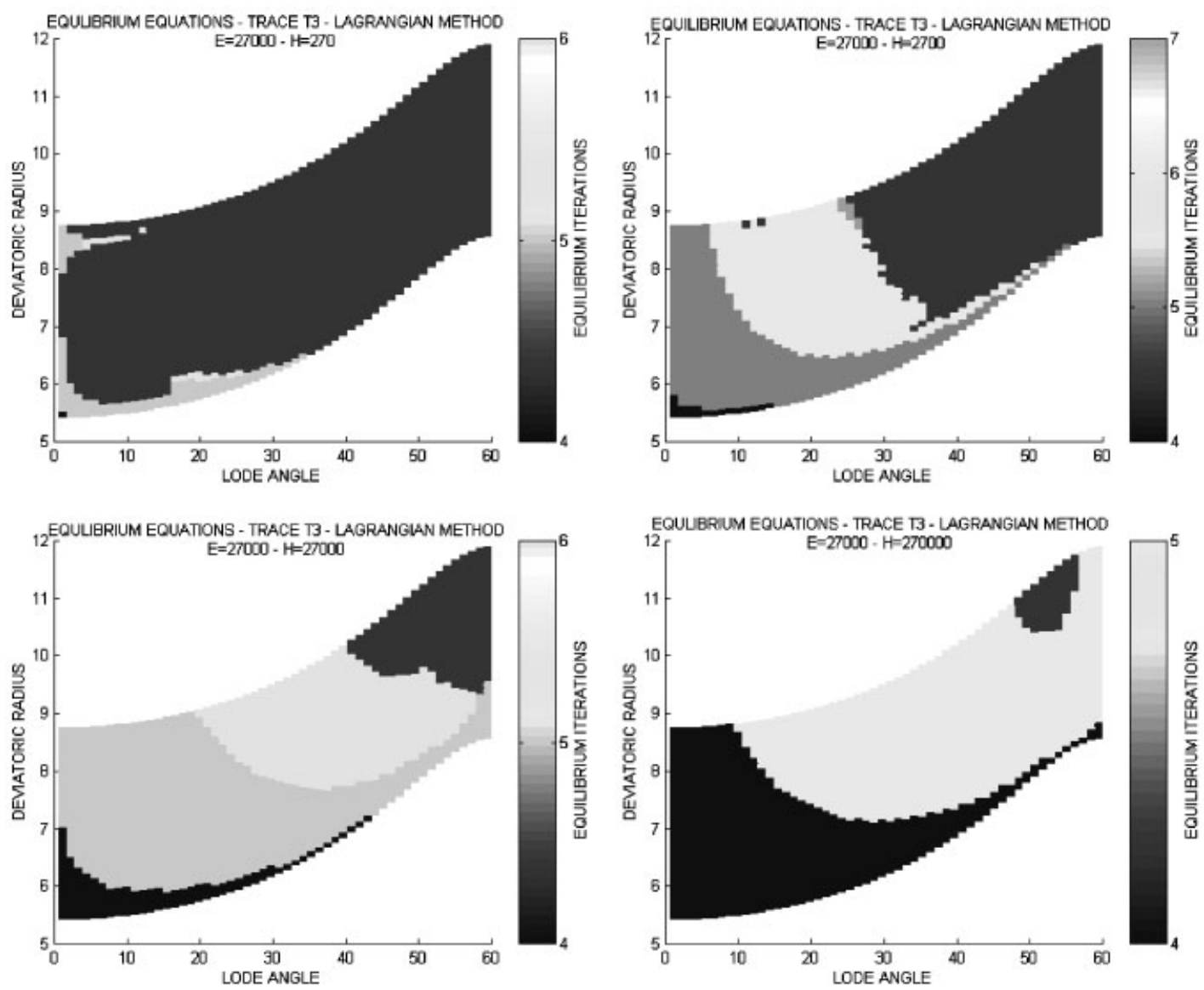

Figure 13. Number of global equilibrium iterations. Lagrangian method.

$\xi=T 3$. Hardening plasticity. Various $H$.

the trial states investigated in Section 4.1.1. It is important to note that all the trial states lie in the ascending branch of the monotonic loading curve, even for damaging plasticity (Figure 12).

Figure 13 reports the number of global equilibrium iterations needed for convergence in the case $\xi=T 3$ in hardening plasticity using the Lagrangian method. It is seen that in this case, even if the local problem always converges, as it is shown in Figure 6, there is lack of convergence of the global equilibrium equations. The dimension of the brown non-convergence region is strongly influenced by the value of the hardening modulus. For the increasing value of $H$ it tends to disappear, vanishing for $H \rightarrow \infty$, when perfect elasticity is attained. For the subsequent applications in hardening plasticity the value $H=2700$ has been used.

Next, a comparison with the Multiplier method is presented. An enlargement of the region of convergence with respect to the Lagrangian method in the sector around $\theta=30^{\circ}$, with a not great increase in the number of iterations, is observed when the method of the multipliers 

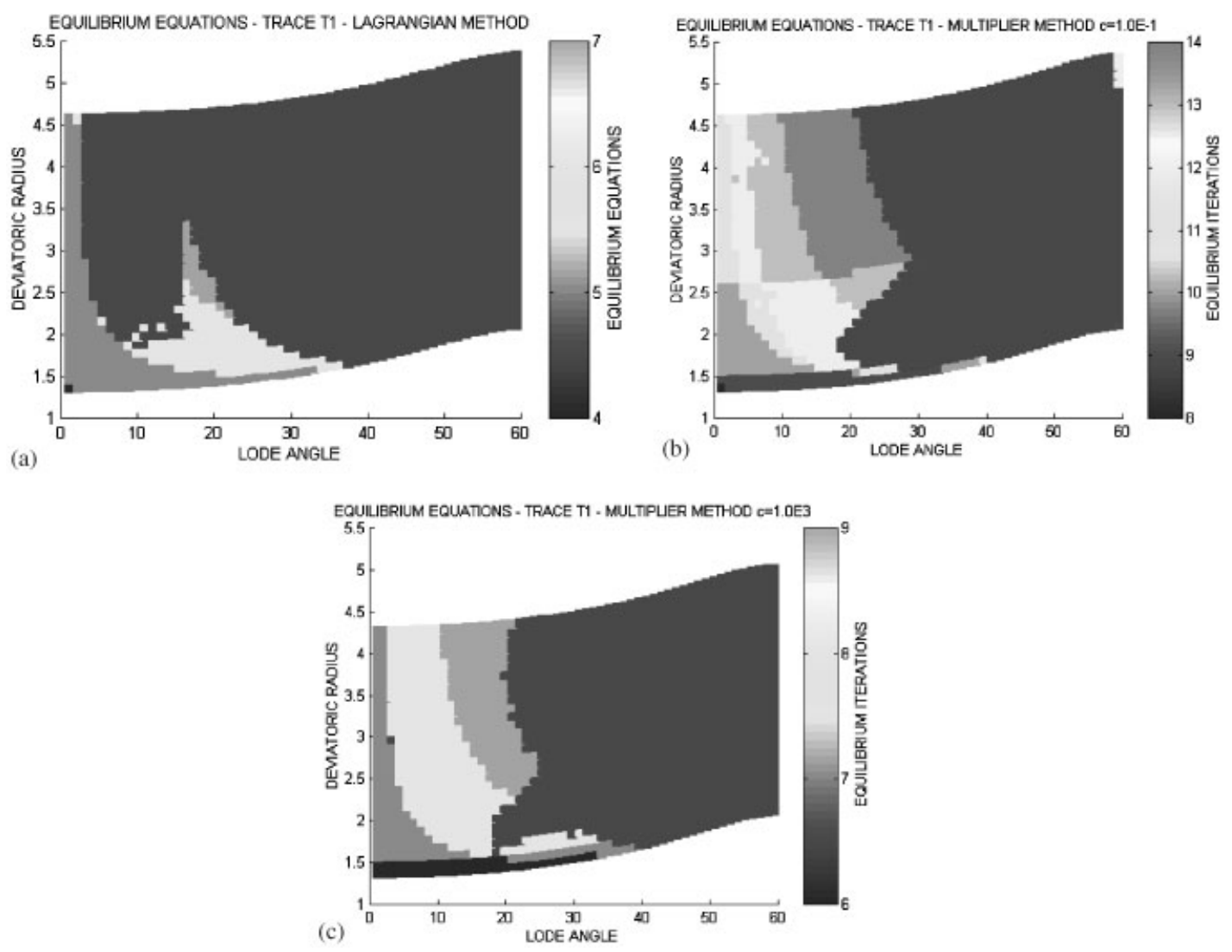

Figure 14. Number of global equilibrium iterations. $\xi=T 1$. Hardening plasticity. $H=2700$ : (a) Lagrangian method; (b) Multiplier method, $c_{0}=10^{-1}$; and (c) Multiplier method, $c_{0}=10^{3}$.

is used. Two values of the initial penalty parameter $c_{0}=10^{-1}$ and $10^{3}$ are used. The cases $\xi=T 1$ and $T 3$ are illustrated in Figures 14 and 15, respectively. Generally, the Multiplier method requires two or three multiplier updates. The penalty parameter $c$ plays an important role in abating the number of iterations. If an initial penalty parameter $c_{0}=10^{3}$ is adopted, an evident reduction in the computational effort is obtained.

Similar considerations are applicable in the case of damaging plasticity, as it is shown in Figures 16 and 17, where a polar representation is utilized. In such cases using the Lagrangian method there is a large region of predictors, close to $\vartheta=60^{\circ}$, where convergence is not reached for a small values of the hardening modulus, i.e. $H=20000$, either in the tension region $(\xi=T 1)$ and in the compression region $(\xi=T 3)$. The performance of the Multiplier method depends strongly on the value of the penalty parameter $c$. In order to enforce convergence, in the numerical applications of the Multiplier method the penalty parameter $c$ has been updated each time the equilibrium convergence is reached, except the first, from the initial value according 

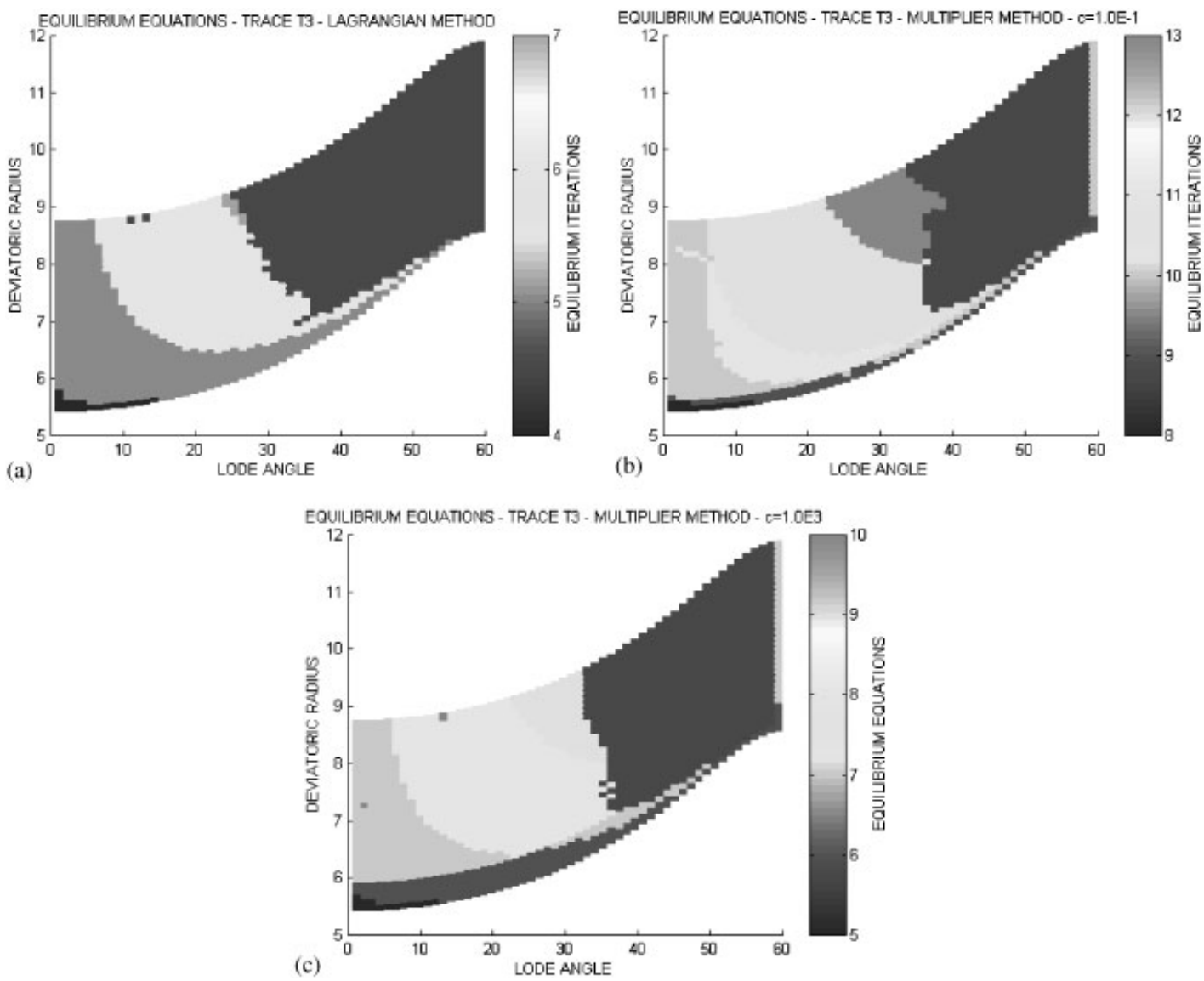

Figure 15. Number of global equilibrium iterations. $\xi=T 3$. Hardening plasticity. $H=2700$ : (a) Lagrangian method; (b) Multiplier method, $c_{0}=10^{-1}$; and (c) Multiplier method, $c_{0}=10^{3}$.

to equation

$$
c_{k+1}=10 c_{k}
$$

until the maximum value $c=10^{6}$ is reached. Using this kind of $c$ update, different from scheme (14), the Multiplier method results converge for almost all the investigated predictors, as shown in Figures 16(c) and 17(c).

\subsection{Multi-surface yield domain}

A second set of results concerns the convergence analysis of an elastic-plastic problem characterized by a yield domain defined as the convex hull of a generalized Drucker-Prager criterion 

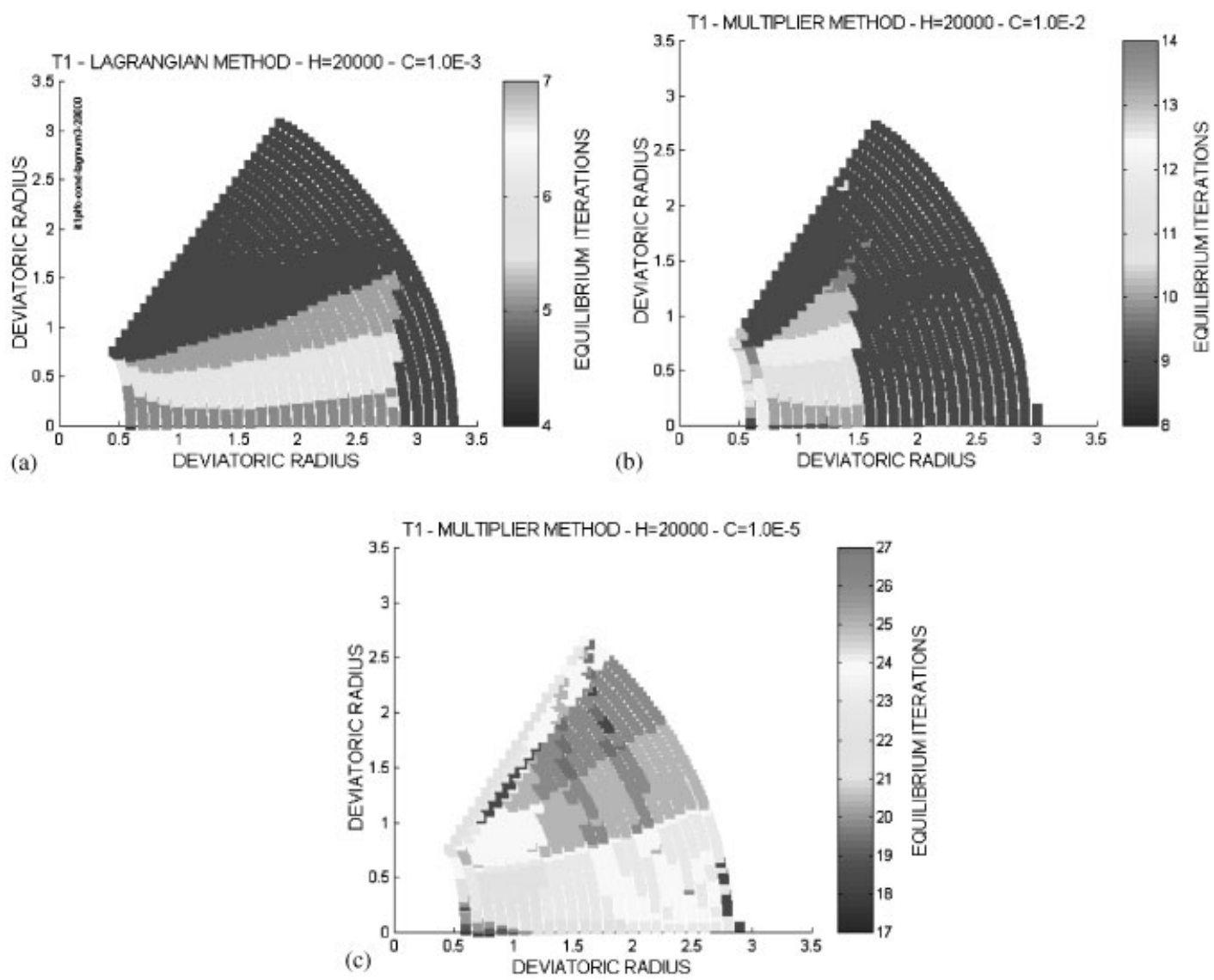

Figure 16. Number of global equilibrium iterations. $\xi=T 1$. Damaging plasticity. $H=20000$ : (a) Lagrangian method; (b) Multiplier method, update formula (53), $c_{0}=10^{-3}$; and (c) Multiplier method, update formula (53) $c_{0}=10^{-5}$.

$g_{1}$ and a pure damage criterion $g_{2}$, i.e. using a combination of the criteria listed below

$$
\begin{aligned}
g_{1} & =\sqrt{3 J_{2}}+\frac{\beta}{3} I_{1}-\left(k_{0}+\chi-k \zeta\right), \quad \beta, k_{0}, k \in R^{+} \\
\beta & =3 \frac{\xi-1}{\xi+1}+3 \frac{k n}{2 E_{0}} \frac{\left|y_{\mathrm{c}}\right|}{\xi} \frac{\left(\xi^{2}-1\right)}{(\xi+1)}, \quad k_{0}=2 \frac{\left|y_{\mathrm{c}}\right|}{(\xi+1)}+\frac{k n}{2 E_{0}} \frac{y_{\mathrm{c}}^{2}}{\xi}, \quad \xi=\frac{\left|y_{\mathrm{c}}\right|}{y_{\mathrm{t}}} \\
g_{2} & =\zeta-\zeta_{0}, \quad \zeta_{0} \in R^{+}
\end{aligned}
$$

Six constitutive parameters completely define the model. They are

$y_{\mathrm{c}}$ compressive strength

$y_{\mathrm{t}}$ tensile strength 

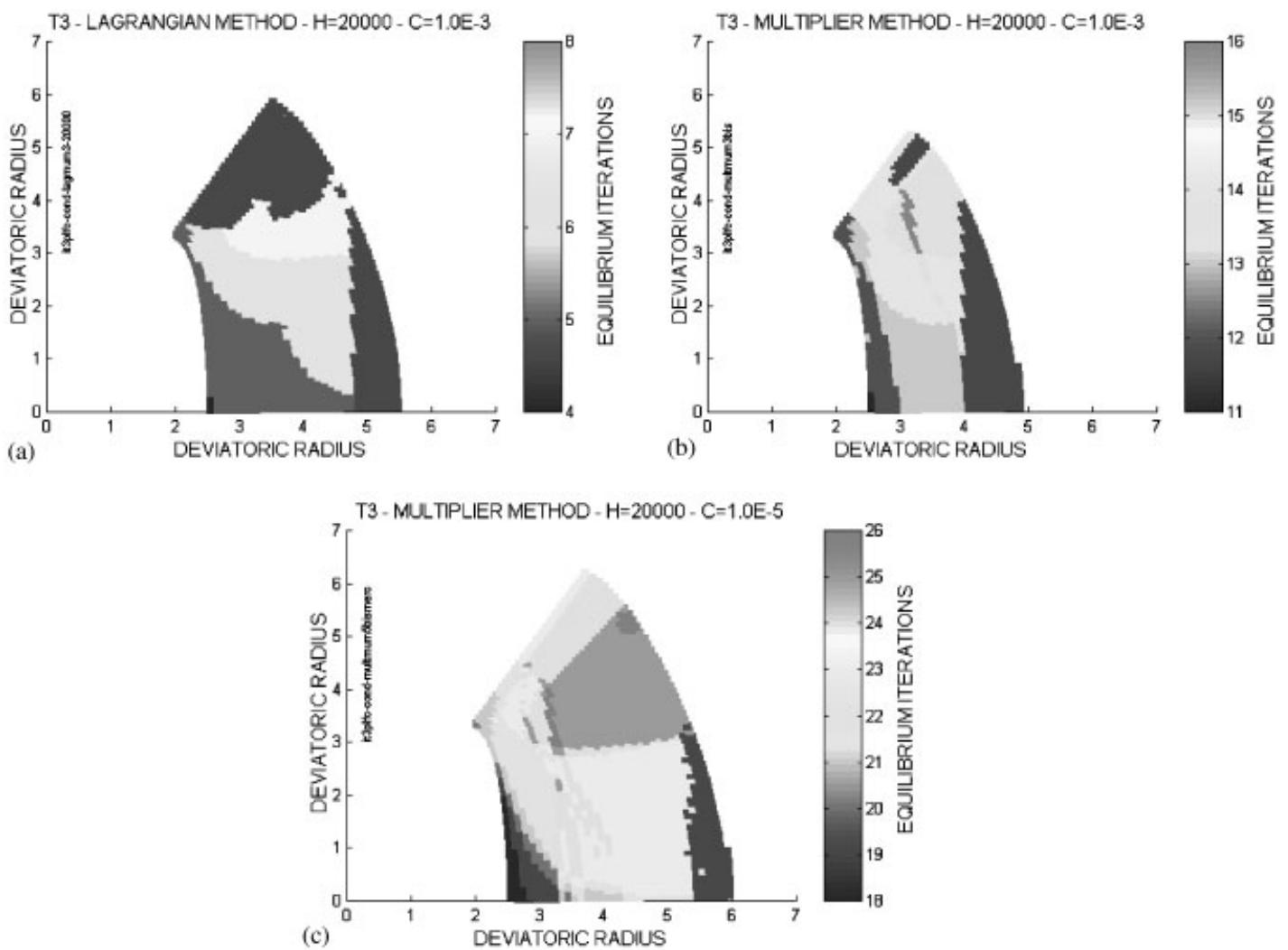

Figure 17. Number of global equilibrium iterations. $\xi=T 3$. Damaging plasticity. $H=20000$ : (a) Lagrangian method; (b) Multiplier method, update formula (53), $c_{0}=10^{-3}$; and (c) Multiplier method, update formula (53), $c_{0}=10^{-5}$.

$E_{0} \quad$ undamaged elastic modulus

$k$ damage rate

$n$ damage exponent

$\zeta_{0}$ fracture energy

Note that also $g_{1}$ depends explicitly on the damage energy $\zeta$.

In this situation the intersection between the two surfaces generates curves where the yield function becomes non-differentiable and numerical troubles arise in the solution of the problem.

The example is only meant as a check of the rate of convergence of the numerical procedures. Therefore, all the problems concerned with the onset of strain localization are out of our considerations.

A homogeneous deformation driven uniaxial compression process is considered, with a constant step-size. The stress-strain curve obtained is represented with a solid line in Figure 18. The evolution of the representative point of the system is sketched in Figure 19 in the plane $g_{1}, g_{2}$. In region A the sample is elastic, then in region B the Drucker-Prager plasticity 


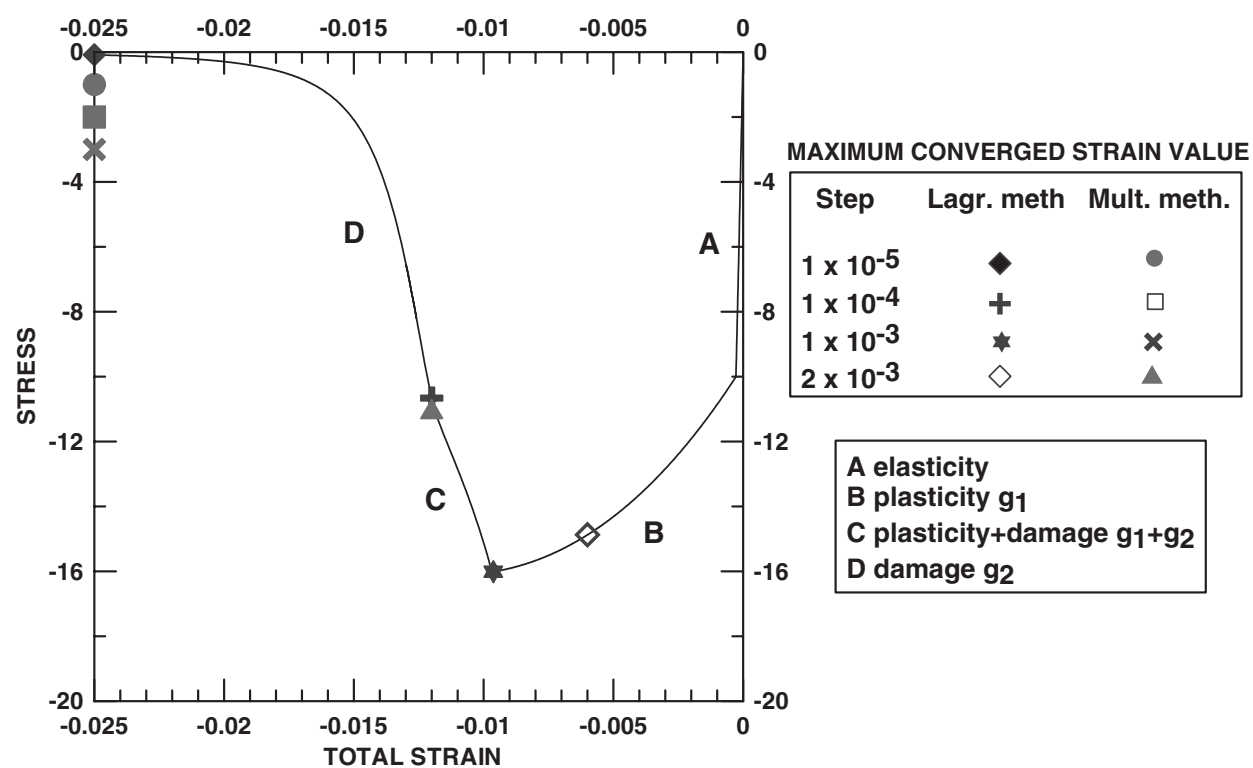

Figure 18. Strain driven uniaxial compression process. Maximum converged displacement for different step-sizes.

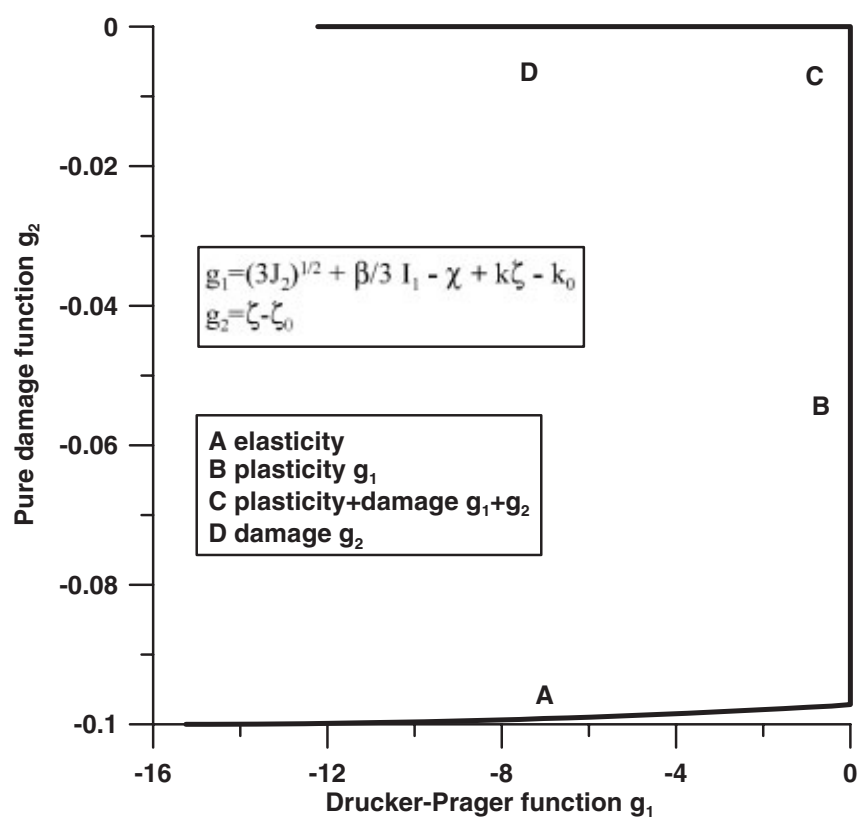

Figure 19. Evolution of the yield functions for the problem of Figure 18. 
criterion (54a) coupled with hardening and damage is activated. Region $\mathrm{C}$ corresponds to a corner point, that is the system evolves while both the plasticity and the damage constraints are active. Finally, region D corresponds to pure damage dissipation mode (54b). Figure 18 also reports the largest step for which convergence is reached, using either the Lagrangian method and the Multiplier method with different step amplitude. It is important to note that convergence of the Lagrangian algorithm is always obtained but a very small step is needed in the descending branch, especially in the region D. Specifically, a step not greater than $10^{-5}$ is required if the Lagrangian method is used in order to follow the entire curve till a nearly vanishing value of the stress, while if the Multiplier method is adopted, the step can be 100 times greater.

\section{CONCLUSIONS}

An analysis of the convergence properties of the non-linear constrained equilibrium problem has been presented that is met in elastic-plastic structural models. With the aim of enlarging the radius of convergence of the standard Lagrangian method, he so-called Multiplier method has been proposed, as an alternative, which is a staggered procedure that has been applied directly to the governing equilibrium equations of the problem. The implementation of the algorithm has been described in Section 3.2; it is quite similar to standard algorithms used in F.E. codes, so that they can be adapted with minor modifications. An efficient version of the Lagrangian method has also been introduced, which turns the inequality admissibility constraint into an equivalent equality one. Through a series of tests it has been found that the Lagrangian method so implemented appears to be able to reach convergence for the local admissibility problem always, even near the region of sharp curvature of the yield function. However, in these cases, the equilibrium equations may diverge, unless a very small step-size is adopted. The Multiplier method substantially enlarges the region of convergence of the equilibrium problem, as it has been shown in Section 4.1.2. It is stressed that the solutions of the equilibrium and compatibility optimization problems were in any case obtained using Newton's method, so that a quadratic rate of convergence was achieved for them, with no apparent need, at least for the cases considered, of introducing line search techniques.

A direct comparison of the two methods, performed through the structural example of Section 4.2, has shown that the Multiplier method allows to use a step-size 100 times greater than the Lagrangian method. Even if the Multiplier method has a linear or super-linear rate of convergence, i.e. it requires more iterations to converge w.r.t. the Lagrangian method, this is more than compensated by the possibility of large step-sizes.

Once the effectiveness of the Multiplier method has been proved, it must be acknowledged that, of course, an optimal strategy would require to combine the two methods, so that one starts with the faster Lagrangian method and switches to the Multiplier method only when convergence is not reached after a reasonable number of iterations; next a switch back to the Lagrangian method, once the trial point has been brought back inside the convergence radius of the Newton's method, could be considered. Such a combined strategy and the switching rules will be considered in a future work. 
A.1. Notes on the use of an augmented Lagrangian functional for the elastic-plastic potential

It has been pointed out in Section 3 that either the Lagrangian or the augmented Lagrangian terms in the functional (25) can be interpreted as regularization of the complementary dissipation potential, equal to the convex indicator function of the yield domain (see formulas (26) and (37)). In other words, the solution $\left(\tau^{*}, \lambda^{*}\right)$ of either problems

$$
\begin{aligned}
\min _{\tau} L(\tau, \lambda)= & \min _{\tau}\left\{\langle\tau, \eta\rangle-e^{\mathrm{c}}(\tau)-\left\langle\tau, \eta_{\mathrm{p}}^{0}\right\rangle-\langle\lambda, \bar{g}(\tau)\rangle\right\} \quad \text { sub. to } \bar{g}(\tau)=0 \\
\min _{\tau} L(\tau, \lambda)= & \min _{\tau}\left\{\langle\tau, \eta\rangle-e^{\mathrm{c}}(\tau)-\left\langle\tau, \eta_{\mathrm{p}}^{0}\right\rangle-\langle\lambda, \bar{g}(\tau)\rangle\right. \\
& \left.-\frac{1}{2} c\langle\bar{g}(\tau), \bar{g}(\tau)\rangle\right\} \quad \text { sub. to } \bar{g}(\tau)=0, \quad c \in R
\end{aligned}
$$

coincide.

The systematic use of augmented Lagrangian functionals in Structural Mechanics was perhaps first introduced in the treatment of unilateral contact problems, with the intent of overcoming the inefficiency of the traditional penalty method in strictly enforcing the one-sided constraints [20-22]. The Lagrangian term, indeed, is effective when the iterative solution point gets closer to the boundary of the constraint, while the penalty term avoids that the tentative solution gets too far from the admissible domain. More recently it has also been used for enforcing boundary conditions in PU methods [14].

Since the augmented Lagrangian functional introduces a real constant $c$, it is the starting point of the Multiplier method, for which the real constant is the step-size of the multiplier update, as it has been pointed out in Section 2.2. It was also noted that the Multiplier method consists in an optimization for the dual functional of $L_{c}$. One might be tempted then to directly use a Newton's iteration simultaneously on both direct variables and Lagrange multipliers, for optimizing the augmented Lagrangian functional. In Reference [4] the authors called this procedure 'Augmented primal closest projection point method'.

We are now going to examine in detail a Newton's iteration for the functional $L_{c}$, in the case of inequality constraint, thus substituting to the function $g$ the equality constraint $\bar{g}$ defined by (2). For simplicity of notation it is considered the case of a single constraint (see Section 3.1 for the case of multiple constraints). The $i$ th iteration is obtained solving the system (all variables have to be intended as $i$ th estimates)

$$
\left[\begin{array}{cc}
\bar{F} & \nabla_{\tau} \bar{g} \\
\nabla_{\tau}^{\mathrm{T}} \bar{g} & \nabla_{\lambda} \bar{g}
\end{array}\right]\left[\begin{array}{c}
\Delta \tau \\
\Delta \lambda
\end{array}\right]=\left[\begin{array}{cl}
\nabla_{\tau} L_{c} \\
-\bar{g}
\end{array}\right], \begin{aligned}
& \bar{F}=E_{\mathrm{t}}^{-1}+(\lambda+c \bar{g}) \nabla_{\tau, \tau}^{2} \bar{g}+c \nabla_{\tau} \bar{g} \otimes \nabla_{\tau} \bar{g} \\
& E_{\mathrm{t}}^{-1}=\nabla_{\tau, \tau}^{2} e^{\mathrm{c}}(\tau) \\
& \nabla_{\tau} L_{c}=\eta-\nabla_{\tau} e^{\mathrm{c}}(\tau)-\eta_{\mathrm{p}}^{0}-(\lambda+c \bar{g}) \nabla_{\tau} \bar{g}
\end{aligned}
$$

The derivatives of the function $\bar{g}$ are given by (32) where the if-conditions has to be intended evaluated at the $i$ th iterate, i.e. whether $g\left(\tau_{i}\right)>-\lambda_{i} / c$ or vice versa. Solving for $\Delta \tau$ from the first row of (A1) and substituting into the second it is found

$$
\Delta \lambda=\left[\nabla_{\tau}^{\mathrm{T}} \bar{g} \bar{F}^{-1} \nabla_{\tau} \bar{g}-\nabla_{\lambda} \bar{g}\right]^{-1}\left[\bar{g}+\nabla_{\tau}^{\mathrm{T}} \bar{g} \bar{F}^{-1} \nabla_{\tau} L_{c}\right]
$$


Inserting the expression of the gradient of $L_{c}$ given in (A1) it is found

$$
\Delta \lambda=\left[\nabla_{\tau}^{\mathrm{T}} \bar{g} \bar{F}^{-1} \nabla_{\tau} \bar{g}-\nabla_{\lambda} \bar{g}\right]^{-1}\left[\left(\bar{g}+\nabla_{\tau}^{\mathrm{T}} \bar{g} \bar{F}^{-1} \dot{\eta}_{\mathrm{p}}\right)-(\lambda+c \bar{g})\left(\nabla_{\tau}^{\mathrm{T}} \bar{g} \bar{F}^{-1} \nabla_{\tau} \bar{g}\right)\right]
$$

having indicated with $\dot{\eta}_{\mathrm{p}}=\eta-\eta_{\mathrm{p}}^{0}-\nabla_{\tau} e^{\mathrm{c}}(\tau)$.

The first row of (A1) becomes then

$$
\Delta \tau=\bar{F}^{-1}\left[\eta-\eta_{\mathrm{p}}^{0}-\nabla_{\tau} e^{\mathrm{c}}(\tau)-(\lambda+c \bar{g}+\Delta \lambda) \nabla_{\tau} \bar{g}\right]=\bar{F}^{-1} \nabla_{\tau} L_{c}\left(\tau_{i}, \lambda_{i+1}, c\right)
$$

We now evaluate explicitly expression (A4). Using (A3) and rearranging one gets

$$
\begin{aligned}
\Delta \tau= & {\left[\bar{F}^{-1}-\bar{F}^{-1} \nabla_{\tau} \bar{g}\left[\nabla_{\tau}^{\mathrm{T}} \bar{g} \bar{F}^{-1} \nabla_{\tau} \bar{g}-\nabla_{\lambda} \bar{g}\right]^{-1} \nabla_{\tau}^{\mathrm{T}} \bar{g} \bar{F}^{-1}\right]\left[\dot{\eta}_{\mathrm{p}}-(\lambda+c \bar{g}) \nabla_{\tau} \bar{g}\right] } \\
& -\bar{F}^{-1} \nabla_{\tau} \bar{g}\left[\nabla_{\tau}^{\mathrm{T}} \bar{g} \bar{F}^{-1} \nabla_{\tau} \bar{g}-\nabla_{\lambda} \bar{g}\right]^{-1} \bar{g}
\end{aligned}
$$

Recalling that

$$
\bar{g}=\left\{\begin{array}{l}
g \\
-\frac{\lambda}{c}
\end{array}, \quad \nabla_{\tau} \bar{g}=\left\{\begin{array}{l}
\nabla_{\tau} g \\
0
\end{array}, \quad \nabla_{\lambda} \bar{g}=\left\{\begin{array}{c}
0 \\
-\frac{1}{c},
\end{array} \quad \nabla^{2} \bar{g}=\left\{\begin{array}{cc}
{\left[\begin{array}{cc}
\nabla_{\tau, \tau}^{2} g & 0 \\
0 & 0
\end{array}\right]} & \text { if } g \geqslant-\frac{\lambda}{c} \\
{\left[\begin{array}{ll}
0 & 0 \\
0 & 0
\end{array}\right]} & \text { if } g<-\frac{\lambda}{c}
\end{array}\right.\right.\right.\right.
$$

expressions (A3) and (A5) specialize, for the cases of active or deactivating constraints, as

$$
\begin{aligned}
& \Delta \lambda= \begin{cases}-(\lambda+c g)+\left[\nabla_{\tau}^{\mathrm{T}} g F^{-1} \nabla_{\tau} g\right]^{-1}\left[g+\nabla_{\tau}^{\mathrm{T}} g F^{-1} \dot{\eta}_{\mathrm{p}}\right] & \text { if } g \geqslant-\frac{\lambda}{c} \\
-\lambda & \text { if } g<-\frac{\lambda}{c}\end{cases} \\
& \Delta \tau= \begin{cases}{\left[F^{-1}-\frac{F^{-1} \nabla_{\tau} g \otimes \nabla_{\tau} g F^{-1}}{F^{-1} \nabla_{\tau} g \cdot \nabla_{\tau} g}\right] \dot{\eta}_{\mathrm{p}}-\frac{F^{-1} \nabla_{\tau} g}{F^{-1} \nabla_{\tau} g \cdot \nabla_{\tau} g} g} & \text { if } g \geqslant-\frac{\lambda}{c} \\
E_{\mathrm{t}}^{-1} \dot{\eta}_{\mathrm{p}} & \text { if } g<-\frac{\lambda}{c}\end{cases}
\end{aligned}
$$

Note that in (A6) and (A7) the matrix $F=E_{\mathrm{t}}^{-1}+(\lambda+c g) \nabla_{\tau, \tau}^{2} g+c \nabla_{\tau} g \otimes \nabla_{\tau} g$ appears.

Several observations can now be made.

The first term in expression (A7) represents the algorithmic tangent stiffness matrix that has to be compared with the exact one and with the one that is yielded by the classical Lagrangian method (i.e. without the augmentation in the functional). The latter has the same form as (A7), provided the matrix $F$ is substituted with the matrix $G=E_{\mathrm{t}}^{-1}+\lambda \nabla_{\tau, \tau}^{2} g$.

This implies that the two methods, while yielding the same result as far as the constrained constitutive problem is concerned, may have different performances in the solution of the equilibrium problem. Note that the matrix $F$ is obtained adding to $G$ a positive definite term. This is one theoretical advantage of the augmentation, since a good choice of the parameter $c$ may make the matrix $G$ always positive definite. 

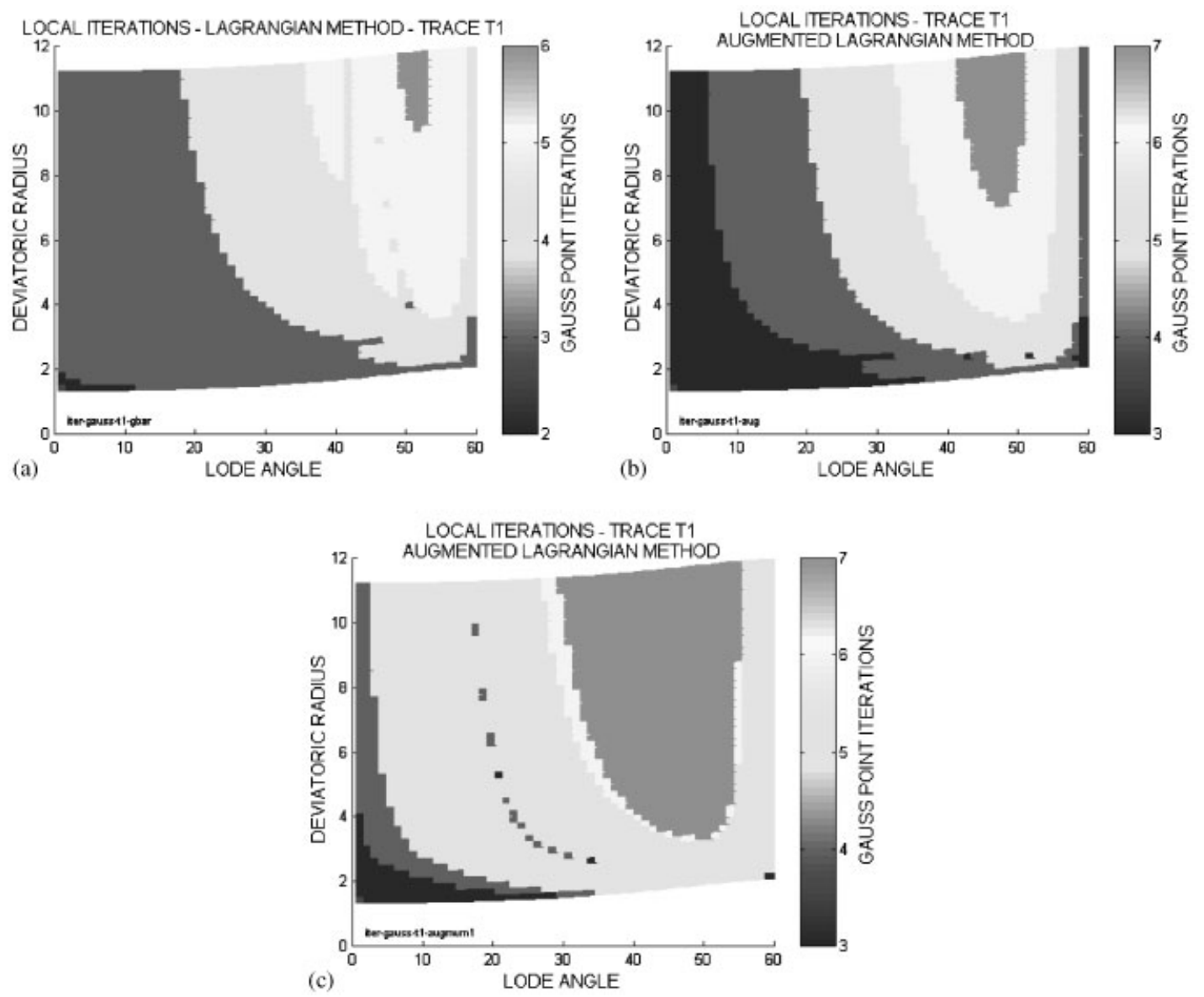

Figure A1. Number of Newton's iterations for the convergence at Gauss point. Perfect plasticity. $\xi=T 1$ : (a) Lagrangian method $c=10^{-4}$; (b) augmented Lagrangian method $c=10^{-4}$; and (c) augmented Lagrangian method $c=10^{-1}$.

According to (A2) and (A4), the iteration can be obtained in two steps:

$$
\begin{aligned}
& \lambda_{i+1}=\lambda_{i}+\Delta \lambda=U\left(\tau_{i}, \lambda_{i}\right) \\
& \tau_{i+1}=\tau_{i}+\Delta \tau=T\left(\tau_{i}, \lambda_{i+1}\right)
\end{aligned}
$$

$U$ and $T$ being two updating formulas given, respectively, by (A6) and (A4).

Update (A8) has the structure of what Tapia defines as diagonalized Multiplier method, which, deriving from a Newton's iteration, presents a locally quadratic rate of convergence [13].

In order to test the effectiveness of the method described, it has been compared with the performance of the Lagrangian method as presented in Section 3.1. 

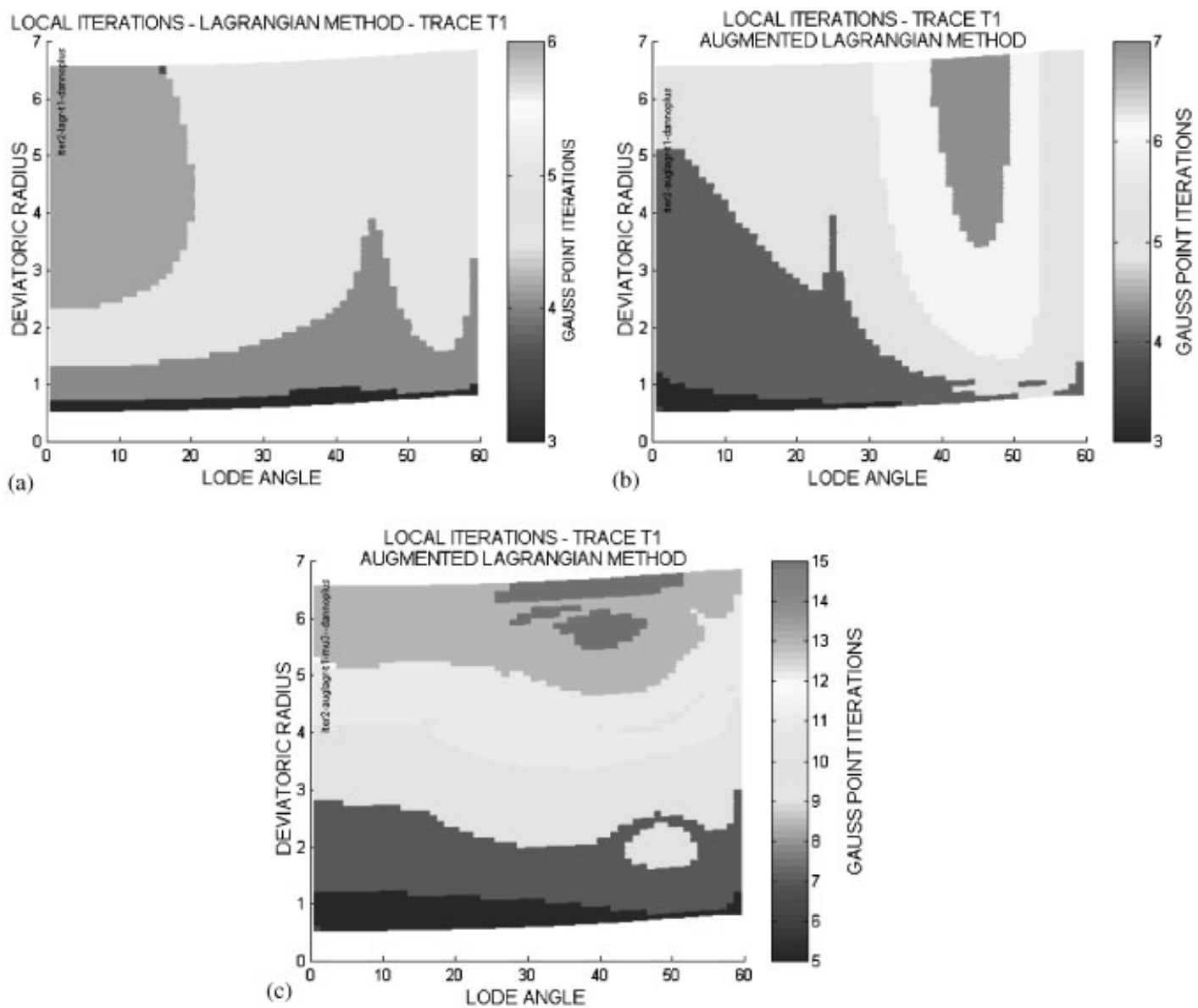

Figure A2. Number of Newton's iterations for the convergence at Gauss point. Damaging plasticity. $\xi=T 1$ : (a) Lagrangian method $c=10^{-4}$; (b) augmented Lagrangian method $c=10^{-4}$; and (c) augmented Lagrangian method $c=10^{3}$.

First, the case of perfect plasticity has been considered. The local constitutive problem has been analysed with the same methodology described in Section 4.1.1, for predictors having hydrostatic component $\xi=T 1$. The results are reported in Figure A1. Figure A1(a) is the same as Figure 6(a), and refers to the Lagrangian method $\left(c=10^{-4}\right)$. Figures A1(b) and A1(c) report the number of iterations needed for convergence using the augmented Lagrangian functional with $c=10^{-4}$ and $10^{-1}$, respectively. While convergence is always reached, it appears that the number of Newton's iterations increases.

Similar results are obtained using a damaging elastic-plastic criterion (introduced in Section 4.1) as shown in Figure A2, where Figure A2(a) coincides with Figure 7(a). These results show the dramatic influence of the value of $c$ on the rate of convergence of the augmented Lagrangian functional. 

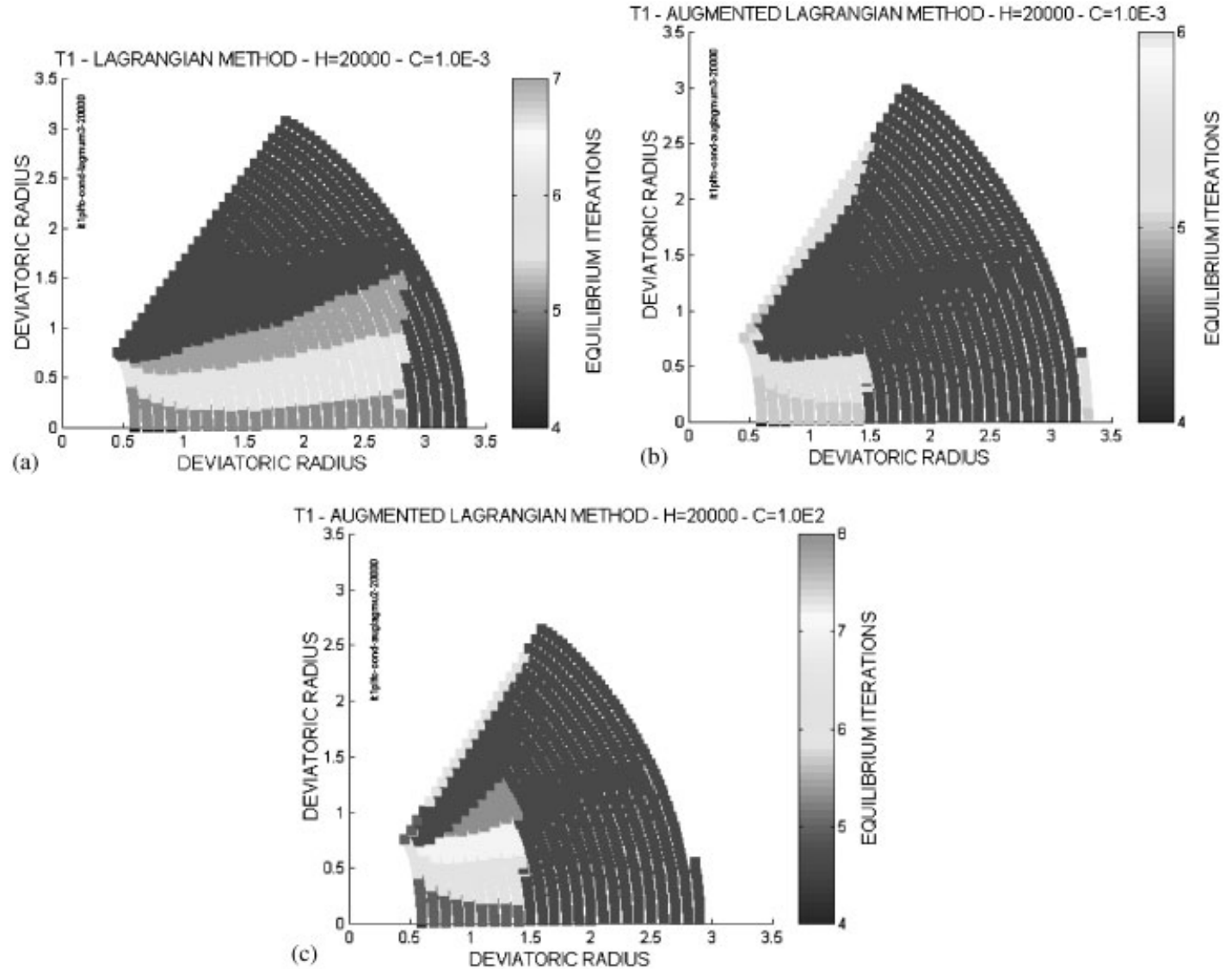

Figure A3. Number of global equilibrium iterations. $\xi=T 1$. Damaging plasticity. $H=20000$ : (a) Lagrangian method $c=10^{-3}$; (b) augmented Lagrangian method $c=10^{-3}$; and (c) augmented Lagrangian method $c=10^{2}$.

Figure A3 presents the number of equilibrium iterations needed for convergence using a damaging criterion, with the same methodology used in Section 4.1.2. Again, the performance of the Lagrangian method is compared with that of the augmented Lagrangian method with two different values of $c$. Apparently, the augmented Lagrangian method performs worse for all the trial states, except for a limited area around the value of the Lode angle $\theta=60^{\circ}$.

The results presented have motivated the fact that the method described in this appendix has not been proposed in the work, since no significant benefit is obtained using the augmented Lagrangian functional in conjunction with primal methods.

\section{REFERENCES}

1. Simo JC, Taylor RL. Consistent tangent operators for rate-independent elasto-plasticity. Computer Methods in Applied Mechanics and Engineering 1985; 48:101-118.

2. Chen WF. Plasticity in Reinforced Concrete. McGraw-Hill: U.S.A., 1982. 
3. Cournier A, He Q-C, Zysset P. Conewise linear elastic materials. Journal of Elasticity 1993; 37(1):1-38.

4. Pérez-Foguet A, Armero F. On the formulation of closest-point projection algorithms in elastoplasticity. Part I: the variational structure. Part II: globally convergent schemes. International Journal for Numerical Methods in Engineering 2000; 53:297-374.

5. Pérez-Foguet A, Rodriguez-Ferran A, Huerta A. Numerical differentiation for local and global tangent operators in computational plasticity. Computer Methods in Applied Mechanics and Engineering 2000; 189:277-296.

6. Runesson K, Samuelsson A, Bernspang L. Numerical technique in plasticity including solution advancement control. International Journal for Numerical Methods in Engineering 1986; 22:769-788.

7. Crouch RS, Tahar B. Application of a stress return algorithm for elasto-plastic hardening-softening models with high yield surface curvature. Proceedings of the European Congress on Computational Methods in Applied Sciences and Engineering ECCOMAS 2000, Barcelona, Spain, 2000, full text in CD ROM.

8. Alfano G, Rosati L, Valoroso N. A tangent-secant approach to rate independent elastoplasticity: formulations and computational issues. Computer Methods in Applied Mechanics and Engineering 1999; 179:379-405.

9. Bertsekas DP. Constrained Optimization and Lagrange Multiplier Methods. Academic Press: Boston, 1982.

10. Hestenes MR. Multiplier and gradient methods. Journal of Optimization Theory and Applications 1969; 4: 303-320.

11. Powell MJ. A method for non linear constraint in optimization problems. In Optimization, Fletcher R (ed.). vol. 4. Academic Press: New York, 1969; 283-298.

12. Buys JD. Dual algorithms for constrained optimisation. Ph.D. Thesis, Rijksununiversiteit de Leiden, The Netherlands, 1972.

13. Tapia RA. Diagonalized multiplier methods and quasi-Newton methods for constrained optimisation. Journal of Optimization Theory and Applications 1977; 22:135-194.

14. Ventura G. An augmented Lagrangian approach to essential boundary conditions in meshless methods. International Journal for Numerical Methods in Engineering 2002; 53:825-842.

15. Contrafatto L, Cuomo M. A new thermodynamically consistent continuum model for hardening plasticity coupled with damage. International Journal of Solids and Structures 2002; 39:6241-6271.

16. Taylor RL. FEAP-A Finite Element Analysis Program. University of California at Berkeley: Berkeley, CA, 2002.

17. Contrafatto L, Cuomo M. Elastic-plastic damaging behaviour of concrete: a generalised Ottosen criterion. In Proceedings of the VII International Conference on Computational Plasticity COMPLAS 2003, Onate E, Owen DRJ (eds). Barcelona, Spain, 2003, CIMNE, full text in CD ROM.

18. Van Mier JGM. Strain softening of concrete under multiaxial loading conditions. Ph.D. Thesis, Eindhoven, 1984.

19. Kupfer H, Gerstle KH. Behavior of concrete under biaxial stresses. Journal of the Engineering Mechanics Division (ASCE) 1973; 99:852-866.

20. Simo J, Laursen T. An Augmented Lagrangian treatment of contact problems involving friction. Computers and Structures 1992; 42(1):97-116.

21. Bille JPh, Cescotto S, Habraken AM, Charlier R. Numerical approach of contact using an Augmented Lagrangian method. In Contact Mechanics, Raous R, Jean M, Moreau JJ (eds). Plenum Press: New York, 1995; 243-246.

22. Cuomo M, Ventura G. Complementary energy approach to contact problems based on consistent Augmented Lagrangian formulation. Mathematical and Computer Modelling 1998; 28(4-8):185-204. 